\title{
Total Synthesis of the Formamicin Aglycon, Formamicinone
}

\author{
Brad M. Savall, Nicolas Blanchard and William R. Roush* \\ Department of Chemistry, University of Michigan, Ann Arbor, MI 48109 \\ Email Address: roush@umich.edu
}

SUPPORTING INFORMATION

Experimental procedures for synthesis of compounds 8 to 16; Stereochemical assignments for compounds 9 and 22; and comparison of the ${ }^{1} \mathrm{H}-\mathrm{NMR}$ and ${ }^{13} \mathrm{C}-\mathrm{NMR}$ data of formamicin and formamicin aglycon (25 pages). This material is available free of charge via the Internet at http://pubs.acs.org. 
General. All reactions were conducted in flame-dried glassware under nitrogen. All solvents were purified before use. THF and $\mathrm{Et}_{2} \mathrm{O}$ were dried by distillation from sodium benzophenone ketyl, or used as is from a solvent purification system (Anhydrous Engineering) ${ }^{1} \cdot \mathrm{CH}_{2} \mathrm{Cl}_{2}$ was dried by distillation from $\mathrm{CaH}_{2}$, or used as is from a solvent purification system (Anhydrous Engineering). Pyridine and 2,6-lutidine were dried by distillation from $\mathrm{CaH}_{2}$. Methanol was distilled from magnesium turnings. Toluene was dried by distillation from sodium. All other commercially available reagents and solvents were used as received. $4 \AA$ molecular sieves were flame-dried under vacuum just prior to use.

${ }^{1} \mathrm{H}$ NMR data were recorded at either $500 \mathrm{MHz}$ or $400 \mathrm{MHz}$ using a Varian I-500 or a Varian XL-400 instrument respectively. ${ }^{1} \mathrm{H}$ NMR chemical shifts are reported relative to residual $\mathrm{CHCl}_{3}(7.26 \mathrm{ppm}) .{ }^{13} \mathrm{C} \mathrm{NMR}$ data were recorded at either $125 \mathrm{MHz}$ or $100 \mathrm{MHz}$ using a Varian I-500 or a Varian XL-400 instrument respectively. ${ }^{13} \mathrm{C}$ chemical shifts are reported relative to the central line of $\mathrm{CDCl}_{3}(77.0 \mathrm{ppm})$. Infrared spectra were recorded using a Perkin Elmer Spectrum 1000 FT-IR (thin film). High resolution mass spectroscopy were performed on a VG 70-250-S Micromass, Inc. mass spectrometer at the University of Michigan Mass Spectrometry Laboratory. Optical rotations were measured on a Rudolph Autopol III polarimeter using a $1 \mathrm{~mL}$ capacity quartz cell with a $10 \mathrm{~cm}$ path length. Elemental analyses were performed by the Elemental Analysis Laboratory at the University of Michigan.

Chromatographic purifications were performed using Kieselgel 60, 230-400 mesh, silica gel unless indicated otherwise. All compounds purified by chromatography

\footnotetext{
${ }^{1}$ Pangborn, A. B.; Giardello, M. A.; Grubbs, R. H.; Rosen, R. K.; Timmers, F. J. Organomet. 1996, 15, 1518.
} 
were sufficiently pure for use in further experiments, unless indicated otherwise. Analytical and semi-preparative HPLC normal phase separations were performed using an HPLC system composed of two Rainin HPXL pumps, a Rheodyne 7125 injector, a Dynamax UV-C or RI-1 detector and Dynamax software on a Macintosh II SI to integrate the peaks. $\mathrm{CuTC},{ }^{2} \mathrm{Ph}_{2} \mathrm{P}(\mathrm{O}) \mathrm{OBu}_{4} \mathrm{~N}^{3}$ and $(E)$-ethyl- $\beta$-iodoacrylate ${ }^{4}$ were prepared according to literature procedures.

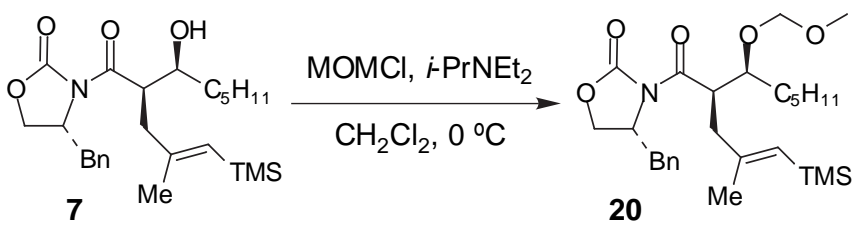

Synthesis of (4R) 4-Benzyl-3-[2-(1'S,2R,4E)-(1'-methoxymethoxy-hexyl)-4-methyl-5(trimethylsilanyl)-pent-4-enoyl]-oxazolidin-2-one (20). To a solution of 7 (15.7 g, 35.3 mmol) and $i$ - $\mathrm{Pr}_{2} \mathrm{NEt}\left(18.5 \mathrm{~mL}, 105.8 \mathrm{mmol}, 3\right.$ eq.) in $\mathrm{CH}_{2} \mathrm{Cl}_{2}(7.5 \mathrm{~mL})$ at $\mathrm{O}{ }^{\circ} \mathrm{C}$ was added dropwise $\mathrm{MOMCl}(7.7 \mathrm{~mL}, 101.0 \mathrm{mmol}, 2.9$ eq. $)$. After $1.5 \mathrm{~h}$ at $0{ }^{\circ} \mathrm{C}$, the reaction mixture was diluted with brine and the aqueous layer extracted with $\mathrm{CH}_{2} \mathrm{Cl}_{2}$. The combined organic layers were dried over $\mathrm{MgSO}_{4}$, filtered and concentrated. The crude residue was taken up in cold hexanes $\left(0^{\circ} \mathrm{C}\right)$, filtered through a pad of celite and rinsed thoroughly with hexanes. After concentration, 20 was obtained as a yellow oil which was used directly in the next step.

Purification of an aliquot by flash chromatography on silica gel (hexanes / ethyl acetate $=85 / 15)$ afforded 20 as a colorless oil having the following properties: ${ }^{1} \mathrm{H}$ NMR

\footnotetext{
${ }^{2}$ Allred, G. D.; Liebeskind, L. S. J. Am. Chem. Soc. 1996, 118, 2748.

${ }^{3}$ Srogl, J.; Allred, G. D.; Liebeskind, L. S. J. Am. Chem. Soc. 1997, 119, 12376.

${ }^{4}$ Dixon, D. J.; Ley, S. V.; Longbottom, D. A. J. Chem. Soc., Perkin Trans. 1 1999, 2231; Hashimoto, N.; Aoyama, T.; Shioiri, T. Chem. Pharm. Bull. 1981, 29, 1475.
} 
$\left(500 \mathrm{MHz}, \mathrm{CDCl}_{3}\right) \delta$ 7.35-7.32 (2H), 7.29-7.26 (1H), $7.22(\mathrm{~d}, J=6.8 \mathrm{~Hz}, 2 \mathrm{H}), 5.27(\mathrm{~s}$, $1 \mathrm{H}), 4.71(\mathrm{dd}, J=7.1 \mathrm{~Hz}, 1 \mathrm{H}), 4.65(\mathrm{ddd}, J=10.0,6.6$ and $2.9 \mathrm{~Hz}, 1 \mathrm{H}), 4.54(\mathrm{~d}, J=7.1$ $\mathrm{Hz}, 1 \mathrm{H}), 4.50(\mathrm{dt}, J=8.8$ and $4.4 \mathrm{~Hz}, 1 \mathrm{H}), 4.09-4.03(2 \mathrm{H}), 3.76(\mathrm{ddd}, J=8.3,4.1$ and 4.1 $\mathrm{Hz}, 1 \mathrm{H}), 3.37(\mathrm{~s}, 3 \mathrm{H}), 3.32(\mathrm{dd}, J=13.8$ and $3.0 \mathrm{~Hz}, 1 \mathrm{H}), 2.65(\mathrm{dd}, J=14.3$ and $10.4 \mathrm{~Hz}$, 1H), $2.57(\mathrm{dd}, J=13.2$ and $10.4 \mathrm{~Hz}, 1 \mathrm{H}), 2.32(\mathrm{dd}, J=13.8$ and $3.8 \mathrm{~Hz}, 1 \mathrm{H}), 1.86$ (s, $3 \mathrm{H}), 1.64-1.47(3 \mathrm{H}), 1.33-1.26(5 \mathrm{H}), 0.89(\mathrm{t}, J=6.8 \mathrm{~Hz}, 3 \mathrm{H}), 0.1(\mathrm{~s}, 9 \mathrm{H}) ;{ }^{13} \mathrm{C}$ NMR (100 $\left.\mathrm{MHz}, \mathrm{CDCl}_{3}\right) \delta 173.6,153.2,152.1,135.5,129.3,128.9,127.3,125.3,96.1,78.8,65.7$, 56.0, 44.0, 40.6, 37.9, 32.0, 31.7, 25.6, 22.6, 22.0, 14.0, 0.0; IR (thin film) 2954, 2360, 1783, 1698, 1382, 1247, 1194, 1038, 840, $700 \mathrm{~cm}^{-1}$; HRMS (FAB) for $\mathrm{C}_{27} \mathrm{H}_{43} \mathrm{O}_{5} \mathrm{SiNa}$ $[\mathrm{M}+\mathrm{Na}]^{+}$calcd. 512.2808, found 512.2816 m/z; Anal. cald for $\mathrm{C}_{27} \mathrm{H}_{43} \mathrm{O}_{5} \mathrm{NSi}$ : C, 66.22; $\mathrm{H}$, 8.85; N, 2.86; Found: C, 66.04; H, 9.08; N, 2.49; $[\alpha]_{\mathrm{D}}{ }^{24}=-81.3\left(c 1.55, \mathrm{CHCl}_{3}\right)$.
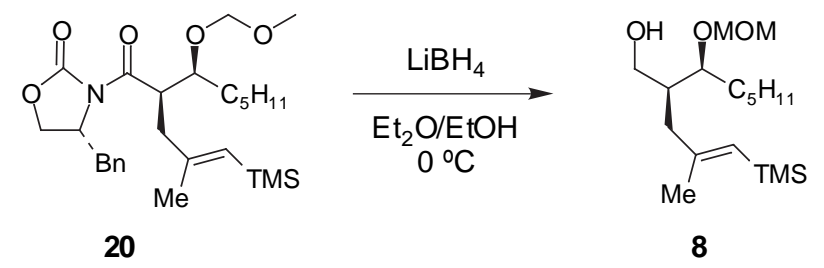

Synthes is of $(1 ' S, 2 S, 4 E) \quad 2-(1$ '-Methoxymethoxy-hexyl)-4-methyl-5(trimethylsilanyl)-pent-4-en-1-ol (8): To a solution of 20 in $\mathrm{Et}_{2} \mathrm{O}(500 \mathrm{~mL})$ and $\mathrm{EtOH}$ (2.45 mL, $42.3 \mathrm{mmol}, 1.2$ eq.) at $0{ }^{\circ} \mathrm{C}$ was added dropwise $\mathrm{LiBH}_{4}(2 \mathrm{M}$ solution in $\mathrm{THF}$, $42.3 \mathrm{mmol}, 21.2 \mathrm{~mL}, 1.2$ eq.). After 4 hours at $0^{\circ} \mathrm{C}$, the reaction mixture was carefully quenched with an aqueous solution of $\mathrm{NaOH}(1 \mathrm{M}, 200 \mathrm{~mL})$ and the solution stirred until clear phases were obtained (1h30). The aqueous layer was extracted with $\mathrm{Et}_{2} \mathrm{O}$, the combined organic layers were washed with brine, dried over $\mathrm{MgSO}_{4}$, filtered and concentrated. Purification of the crude material by flash chromatography on silica gel (hexanes $/$ ethyl acetate $=95 / 5$ to $80 / 20)$ afforded $9.60 \mathrm{~g}(86 \%$ from 7 ) of $\mathbf{8}$ as a colorless 
oil. ${ }^{1} \mathrm{H}$ NMR (400 MHz, $\left.\mathrm{CDCl}_{3}\right) \delta 5.24(\mathrm{~s}, 1 \mathrm{H}), 4.67(\mathrm{~d}, J=6.8 \mathrm{~Hz}, 1 \mathrm{H}), 4.63(\mathrm{~d}, J=6.8$

$\mathrm{Hz}, 1 \mathrm{H}), 3.65$ (m, 1H), $3.63(\mathrm{~m}, 1 \mathrm{H}), 3.55(\mathrm{~m}, 1 \mathrm{H}), 3.40(\mathrm{~s}, 3 \mathrm{H}), 2.81(\mathrm{~m}, 1 \mathrm{H}), 2.11-2.04$ (2H), $1.94(\mathrm{~m}, 1 \mathrm{H}), 1.78(\mathrm{~s}, 3 \mathrm{H}), 1.54(\mathrm{~m}, 1 \mathrm{H}), 1.47-1.42(2 \mathrm{H}), 1.33-1.24(5 \mathrm{H}), 0.88(\mathrm{t}, J$ $=7.0 \mathrm{~Hz}, 3 \mathrm{H}), 0.08(\mathrm{~s}, 9 \mathrm{H}) ;{ }^{13} \mathrm{C} \mathrm{NMR}\left(100 \mathrm{MHz}, \mathrm{CDCl}_{3}\right) \delta 152.8,126.0,96.5,79.6$, 63.5, 55.9, 40.6 (2C), 31.8, 30.4, 25.7, 22.6, 21.1, 14.0, -0.1; IR (thin film) 3445, 2954, $2932,1615,1247,1152,1097,1040,861,837 \mathrm{~cm}^{-1}$; HRMS (CI, $\mathrm{NH}_{3}$ ) for $\mathrm{C}_{17} \mathrm{H}_{36} \mathrm{O}_{3} \mathrm{Si}$ $[\mathrm{M}+\mathrm{H}]^{+}$calcd. 317.2512, found 317.2500 m/z; Anal. cald for $\mathrm{C}_{17} \mathrm{H}_{36} \mathrm{O}_{3} \mathrm{Si}: \mathrm{C}, 64.50 ; \mathrm{H}$, 11.46; Found: C, 64.81; H, 11.08; $[\alpha]_{\mathrm{D}}^{24}=+20.1\left(\right.$ c 1.51, $\left.\mathrm{CHCl}_{3}\right)$.
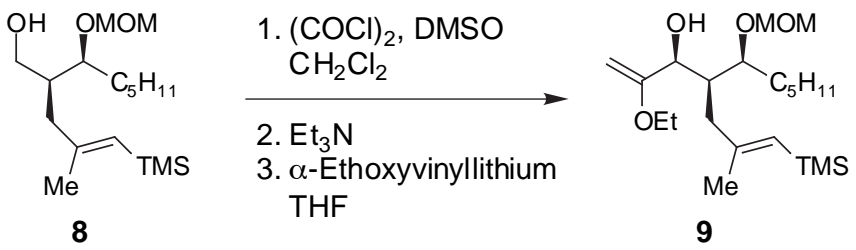

\section{Synthesis of (1'S,3S,4R,6E) 2-Ethoxy-4-(1'-methoxymethoxy-hexyl)-6-methyl-7-} (trimethylsilanyl)-hepta-1,6-dien-3-ol (9): To a solution of oxalyl chloride $(4.3 \mathrm{~mL}$, $49.6 \mathrm{mmol}, 2$ eq.) in $\mathrm{CH}_{2} \mathrm{Cl}_{2}(250 \mathrm{~mL})$ at $-70^{\circ} \mathrm{C}$ was added dropwise DMSO $(5.3 \mathrm{~mL}$, $74.4 \mathrm{mmol}, 3$ eq.). After $15 \mathrm{~min}$. at $-70{ }^{\circ} \mathrm{C}$, a solution of $\mathbf{8}(7.84 \mathrm{~g}, 24.8 \mathrm{mmol})$ in $\mathrm{CH}_{2} \mathrm{Cl}_{2}$ $(15 \mathrm{~mL})$ was added slowly via canula. The reaction mixture was stirred for another 15 min at $-70{ }^{\circ} \mathrm{C}$ before addition of $\mathrm{Et}_{3} \mathrm{~N}(13.8 \mathrm{~mL}, 99.2 \mathrm{mmol}, 4$ eq.). The reaction mixture was allowed to warm to $0{ }^{\circ} \mathrm{C}$ over a $35 \mathrm{~min}$. period and then stirred at $0{ }^{\circ} \mathrm{C}$ for $15 \mathrm{~min}$. $300 \mathrm{~mL}$ of cold hexanes were then added and the resulting suspension was filtered through celite, rinse thoroughly with cold hexanes and concentrated to $150 \mathrm{~mL}$. The solution was diluted with water $(100 \mathrm{~mL})$, and the aqueous layer was extracted with $\mathrm{CH}_{2} \mathrm{Cl}_{2}$. The combined organic layers were washed with brine, dried over $\mathrm{MgSO}_{4}$, filtered and concentrated to give a slightly yellow oil which was used directly in the next step without any purification. To a solution of ethylvinyl ether $(12 \mathrm{~mL}, 125 \mathrm{mmol})$ in 
THF $(25 \mathrm{~mL})$ at $-70{ }^{\circ} \mathrm{C}$ was added $t$-BuLi (1.5 M in pentane, $\left.125 \mathrm{mmol}, 83 \mathrm{~mL}\right)$ dropwise such as $\mathrm{T}<-60^{\circ} \mathrm{C}$. The reaction mixture was then warmed to $0{ }^{\circ} \mathrm{C}$, stirred at that temperature for $30 \mathrm{~min}$, cooled to $-70{ }^{\circ} \mathrm{C}$ and diluted with $\mathrm{THF}(50 \mathrm{~mL})$. The solution of $\alpha$-ethoxyvinyllithium was further cooled to $-118^{\circ} \mathrm{C}$ (internal temperature) and a solution of the above crude aldehyde was then slowly added. After 10 min at -118 ${ }^{\circ} \mathrm{C}$, the reaction mixture was quenched by slow addition of $\mathrm{MeOH}(5 \mathrm{~mL})$ followed by an aqueous saturated solution of $\mathrm{NaHCO}_{3}$. The aqueous layer was extracted with $\mathrm{CH}_{2} \mathrm{Cl}_{2}$ and the combined organic layers washed with brine, dried over $\mathrm{K}_{2} \mathrm{CO}_{3}$, filtered and concentrated to give a slightly yellow oil. Purification by flash chromatography on silica gel (hexanes / ethyl acetate $=95 / 5)$ gave 9 as a colorless oil. ${ }^{1} \mathrm{H}$ NMR $\left(500 \mathrm{MHz}, \mathrm{CDCl}_{3}\right)$ $\delta 5.28(\mathrm{~s}, 1 \mathrm{H}), 4.65(\mathrm{~s}, 2 \mathrm{H}), 4.27(\mathrm{t}, J=3.7 \mathrm{~Hz}, 1 \mathrm{H}), 4.22(\mathrm{~d}, J=1.7 \mathrm{~Hz}, 1 \mathrm{H}), 4.01(\mathrm{~d}, J$ $=1.7 \mathrm{~Hz}, 1 \mathrm{H}), 3.78-3.68(2 \mathrm{H}), 3.63(\mathrm{dt}, J=6.7$ and $2.7 \mathrm{~Hz}, 1 \mathrm{H}), 3.38(\mathrm{~s}, 3 \mathrm{H}), 2.70(\mathrm{~d}, J$ $=4.2 \mathrm{~Hz}, 1 \mathrm{H}), 2.31(\mathrm{dd}, J=13.9$ and $7.6 \mathrm{~Hz}, 1 \mathrm{H}), 2.25(\mathrm{~m}, 1 \mathrm{H}), 2.18(\mathrm{dd}, J=13.9$ and $7.6 \mathrm{~Hz}, 1 \mathrm{H}), 1.75(\mathrm{~s}, 3 \mathrm{H}), 1.62-1.53(2 \mathrm{H}), 1.35-1.17(9 \mathrm{H}$ including $1.27(\mathrm{t}, J=7.1 \mathrm{~Hz}$, $3 \mathrm{H})), 0.87(\mathrm{t}, J=7.1 \mathrm{~Hz}, 3 \mathrm{H}), 0.07(\mathrm{~s}, 9 \mathrm{H}) ;{ }^{13} \mathrm{C} \mathrm{NMR}\left(125 \mathrm{MHz}, \mathrm{CDCl}_{3}\right) \delta 162.7,154.9$, $124.7,95.8,81.7,81.0,74.3,62.7,55.7,40.9,37.0,31.8,31.3,26.0,22.7,21.2,14.4$, 14.0, -0.1; IR (thin film) 3468 (br), 2955, 2361, 1662, 1616, 1247, 1100, 1040, 870, 840 $\mathrm{cm}^{-1}$; HRMS (CI, $\mathrm{NH}_{3}$ ) for $\mathrm{C}_{21} \mathrm{H}_{42} \mathrm{O}_{4} \mathrm{Si}[\mathrm{M}+\mathrm{H}]^{+}$calcd. 387.2931, found 387, $2985 \mathrm{~m} / \mathrm{z}$; $[\alpha]_{\mathrm{D}}^{25}=-3.3\left(c 1.04, \mathrm{CHCl}_{3}\right)$.

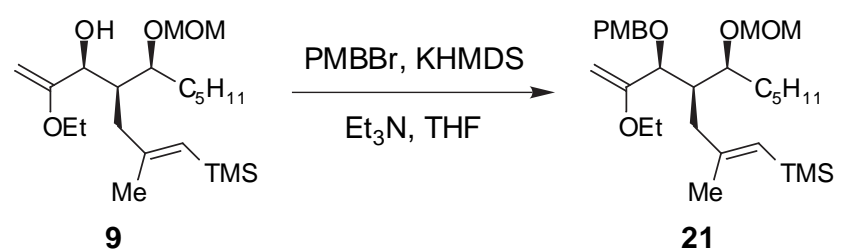

Synthesis of (1'S,3S,4R,6E) 2-Ethoxy-3-p-methoxybenzyloxy-4-(1'-methoxymethoxyhexyl)-6-methyl-7-(trimethylsilanyl)-hepta-1,6-diene (21): To a $0{ }^{\circ} \mathrm{C}$ solution of 9 in 
THF (250 mL) was added $\mathrm{Et}_{3} \mathrm{~N}$ (10.5 mL, 75.0 mmol, 3 eq.), PMBBr (6.9 mL, 2 eq.) followed by $\mathrm{KHMDS}(0.5 \mathrm{M}$ in toluene, $50 \mathrm{~mL}, 1$ eq. $)$. After $1 \mathrm{~h}$ at $0{ }^{\circ} \mathrm{C}$, the reaction mixture was quenched by the addition of an aqueous saturated solution of $\mathrm{NaHCO}_{3}$. The aqueous layer was extracted with ethyl acetate and the combined organic layers washed with brine, dried over $\mathrm{MgSO}_{4}$, filtered and concentrated. Purification of the crude material by flash chromatography on silica gel (hexanes / ethyl acetate $=100 / 3+1 \%$ $\left.\mathrm{NEt}_{3}\right)$ afforded $9.04 \mathrm{~g}(72 \%)$ of 21 as a colorless oil. ${ }^{1} \mathrm{H} \mathrm{NMR}\left(500 \mathrm{MHz}, \mathrm{CDCl}_{3}\right) \delta 7.27$ $(\mathrm{d}, J=8.7 \mathrm{~Hz}, 2 \mathrm{H}), 6.88(\mathrm{~d}, J=8.7 \mathrm{~Hz}, 2 \mathrm{H}), 5.25(\mathrm{~s}, 1 \mathrm{H}), 4.70(\mathrm{~d}, J=6.8 \mathrm{~Hz}, 1 \mathrm{H}), 4.59$ $(\mathrm{d}, J=6.8 \mathrm{~Hz}, 1 \mathrm{H}), 4.55(\mathrm{~d}, J=11.0 \mathrm{~Hz}, 1 \mathrm{H}), 4.30(\mathrm{~d}, J=11.0 \mathrm{~Hz}, 1 \mathrm{H}), 4.20(\mathrm{~d}, J=1.8$ $\mathrm{Hz}, 1 \mathrm{H}), 4.13(\mathrm{~d}, J=1.8 \mathrm{~Hz}, 1 \mathrm{H}), 3.95(\mathrm{~d}, J=3.9 \mathrm{~Hz}, 1 \mathrm{H}), 3.82(\mathrm{~s}, 3 \mathrm{H}), 3.81-3.76(2 \mathrm{H})$, $3.52(\mathrm{dt}, J=8.8$ and $3.4 \mathrm{~Hz}, 1 \mathrm{H}), 3.39(\mathrm{~s}, 3 \mathrm{H}), 2.45(\mathrm{dd}, J=13.9$ and $3.4 \mathrm{~Hz}, 1 \mathrm{H}), 2.29$ (m, 1H), $2.14(\mathrm{dd}, J=13.9$ and $10.6 \mathrm{~Hz}, 1 \mathrm{H}), 1.77(\mathrm{~s}, 3 \mathrm{H}), 1.66(\mathrm{~m}, 1 \mathrm{H}), 1.50-1.41(2 \mathrm{H})$, 1.35-1.20 (8H including $1.32(\mathrm{t}, J=7.0 \mathrm{~Hz}, 3 \mathrm{H})), 0.91(\mathrm{t}, J=7.1 \mathrm{~Hz}, 3 \mathrm{H}), 0.10(\mathrm{~s}, 9 \mathrm{H})$; ${ }^{13} \mathrm{C} \mathrm{NMR}\left(125 \mathrm{MHz}, \mathrm{CDCl}_{3}\right) \delta 160.5,158.9,154.3,130.9,129.2,125.1,113.5,96.1$, 82.7, 78.7, 78.5, 70.3, 62.7, 55.5, 55.2, 41.1, 38.9, 31.7, 30.8, 26.3, 22.7, 21.0, 14.4, 14.1, 0.0; IR (thin film) 2954, 1615, 1515, 1248, 1098, 1038, 869, $837 \mathrm{~cm}^{-1}$; HRMS (FAB) for $\mathrm{C}_{29} \mathrm{H}_{50} \mathrm{O}_{5} \mathrm{SiNa}[\mathrm{M}+\mathrm{Na}]^{+}$calcd. 529.3325, found 529.3322; $[\alpha]_{\mathrm{D}}{ }^{25}=-73.7\left(c\right.$ 1.38, $\left.\mathrm{CHCl}_{3}\right)$.

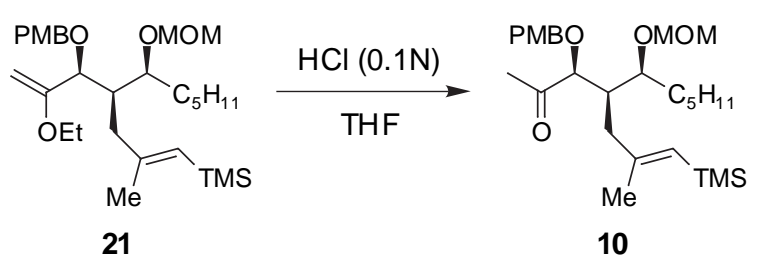

\section{Synthesis of (1'S,3S,4R,6E) 3-p-Methoxybenzyloxy-4-(1'-methoxymethoxy-} hexyl)-6-methyl-7-(trimethylsilanyl)-hept-6-en-2-one (10): To a solution of 21 (7.69 g, $15.18 \mathrm{mmol})$ in THF $(70 \mathrm{~mL})$ at $23{ }^{\circ} \mathrm{C}$ was added an aqueous solution of $\mathrm{HCl}(1 \mathrm{~N}, 70$ $\mathrm{mL}$ ). After $20 \mathrm{~min}$, the aqueous layer was extracted with $\mathrm{CH}_{2} \mathrm{Cl}_{2}$. The combined organic 
layers were washed successively with an aqueous saturated solution of $\mathrm{NaHCO}_{3}$ and brine, dried over $\mathrm{MgSO}_{4}$, filtered and concentrated to give $7.05 \mathrm{~g}(97 \%)$ of a $\mathbf{1 0}$ as a colorless oil. ${ }^{1} \mathrm{H}$ NMR (400 MHz, $\left.\mathrm{CDCl}_{3}\right) \delta 7.21(\mathrm{~d}, J=8.8 \mathrm{~Hz}, 2 \mathrm{H}), 6.84(\mathrm{~d}, J=8.8 \mathrm{~Hz}$, 2H), $5.20(\mathrm{~s}, 1 \mathrm{H}), 4.62(\mathrm{~d}, J=6.6 \mathrm{~Hz}, 1 \mathrm{H}), 4.50(\mathrm{~d}, J=6.6 \mathrm{~Hz}, 1 \mathrm{H}), 4.42(\mathrm{~d}, J=11.0$ $\mathrm{Hz}, 1 \mathrm{H}), 4.27$ (d, $J=11.0 \mathrm{~Hz}, 1 \mathrm{H}), 3.86(\mathrm{~d}, J=4.4 \mathrm{~Hz}, 1 \mathrm{H}), 3.76$ (s, 3H), 3.46 (dt, $J=$ 9.2 and $2.9 \mathrm{~Hz}, 1 \mathrm{H}), 3.33(\mathrm{~s}, 3 \mathrm{H}), 2.38-2.28(2 \mathrm{H}), 2.12(\mathrm{~s}, 3 \mathrm{H}), 2.03(\mathrm{dd}, J=13.4$ and 9.0 $\mathrm{Hz}, 1 \mathrm{H}), 1.69$ (s, 3H), 1.54-1.36 (3H), 1.27-1.14 (5H), 0.84 (t, J = 7.0 Hz, 3H), 0.03 (s, $9 \mathrm{H}) ;{ }^{13} \mathrm{C} \mathrm{NMR}\left(100 \mathrm{MHz}, \mathrm{CDCl}_{3}\right) \delta 211.3,159.3,152.3,129.7,129.4,129.6,113.7$, $96.2,84.3,78.0,72.1,55.8,42.4,39.8,31.6,30.0,26.5,26.2,22.6,21.0,14.0,-0.1$; IR (thin film) 2954, 1715, 1614, 1515, 1249, 1035, $838 \mathrm{~cm}^{-1}$; HRMS (electrospray) for $\mathrm{C}_{27} \mathrm{H}_{46} \mathrm{O}_{5} \mathrm{SiNa}[\mathrm{M}+\mathrm{Na}]^{+}$calcd. 501.3012, found 501.3020; $[\alpha]_{\mathrm{D}}^{25}=-83.0\left(c 0.43, \mathrm{CHCl}_{3}\right)$.

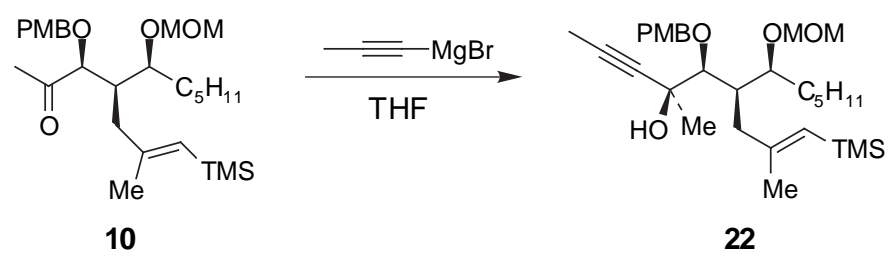

Synthesis of $\quad(1 ' S, 4 S, 5 S, 6 R, 8 E) 5-p$-Methoxybenzyloxy-6-(1'methoxymethoxy-hexyl)-4,8-dimethyl-9-(trimethylsilanyl)-non-8-en-2-yn-4-ol (22): To a solution of $\mathbf{1 0}(4.3 \mathrm{~g}, 9.0 \mathrm{mmol})$ in $\mathrm{THF}(80 \mathrm{~mL})$ at $-45^{\circ} \mathrm{C}$ was added 1propynylmagnesium bromide (0.5 M in THF, $25 \mathrm{mmol}, 50 \mathrm{~mL}, 2.8$ eq.). After $12 \mathrm{~h}$ at $-45^{\circ} \mathrm{C}$, the reaction mixture was allowed to warm to $-30{ }^{\circ} \mathrm{C}$ and stirred $3 \mathrm{~h}$ at that temperature. The reaction was then poured into an aqueous saturated solution of $\mathrm{NH}_{4} \mathrm{Cl}$ and the aqueous layer extracted with $\mathrm{CH}_{2} \mathrm{Cl}_{2}$. The combined organic layers were dried over $\mathrm{MgSO}_{4}$, filtered and concentrated. Purification of the crude material by flash chromatography on silica gel (hexanes / ethyl acetate $=85 / 15$ to $70 / 30$ ) afforded $4.36 \mathrm{~g}$ (94\%) of 22 as a colorless oil. ${ }^{1} \mathrm{H}$ NMR $\left(500 \mathrm{MHz}, \mathrm{CDCl}_{3}\right) \delta 7.27(\mathrm{~d}, J=8.8 \mathrm{~Hz}, 2 \mathrm{H})$, 
$6.87(\mathrm{~d}, J=8.8 \mathrm{~Hz}, 2 \mathrm{H}), 5.24(\mathrm{~s}, 1 \mathrm{H}), 4.73(\mathrm{~d}, J=6.8 \mathrm{~Hz}, 1 \mathrm{H}), 4.70(\mathrm{~d}, J=10.7 \mathrm{~Hz}, 1 \mathrm{H})$, $4.57(\mathrm{~d}, J=6.8 \mathrm{~Hz}, 1 \mathrm{H}), 4.54(\mathrm{~d}, J=10.7 \mathrm{~Hz}, 1 \mathrm{H}), 4.30(\mathrm{~s}, 1 \mathrm{H}), 3.80(\mathrm{~s}, 3 \mathrm{H}), 3.66(\mathrm{~m}$, 1H), $3.45(\mathrm{~d}, J=5.1 \mathrm{~Hz}, 1 \mathrm{H}), 3.39(\mathrm{~s}, 3 \mathrm{H}), 2.68(\mathrm{dd}, J=13.7$ and $3.8 \mathrm{~Hz}, 1 \mathrm{H}), 2.56(\mathrm{~m}$, 1H), $2.00(\mathrm{dd}, J=13.7$ and $11.8 \mathrm{~Hz}, 1 \mathrm{H}), 1.83(\mathrm{~s}, 3 \mathrm{H}), 1.79(\mathrm{~s}, 3 \mathrm{H}), 1.62-1.47(5 \mathrm{H}$ including $1.52(\mathrm{~s}, 3 \mathrm{H})), 1.32-1.23(6 \mathrm{H}), 0.90(\mathrm{t}, J=7.1 \mathrm{~Hz}, 3 \mathrm{H}), 0.10(\mathrm{~s}, 9 \mathrm{H}) ;{ }^{13} \mathrm{C}$ NMR $\left(125 \mathrm{MHz}, \mathrm{CDCl}_{3}\right) \delta 159.1,153.5,130.6,129.3,129.5,113.7,95.2,83.6,82.5,80.2$, 77.6, 74.4, 71.6, 55.9, 55.2, 41.1, 40.4, 31.6, 29.6, 27.3, 26.4, 22.7, 20.8, 14.1, 3.8, -0.1; IR (thin film) 3409 (br), 2954, 2252, 1614, 1515, 1456, 1249, 1095, 1036, 869, $840 \mathrm{~cm}^{-1}$; HRMS (electrospray) for $\mathrm{C}_{30} \mathrm{H}_{50} \mathrm{O}_{5} \mathrm{SiNa}[\mathrm{M}+\mathrm{Na}]^{+}$calcd. 541.3325, found 541.3315; $[\alpha]_{\mathrm{D}}^{25}=-87.9\left(c 0.33, \mathrm{CHCl}_{3}\right)$.

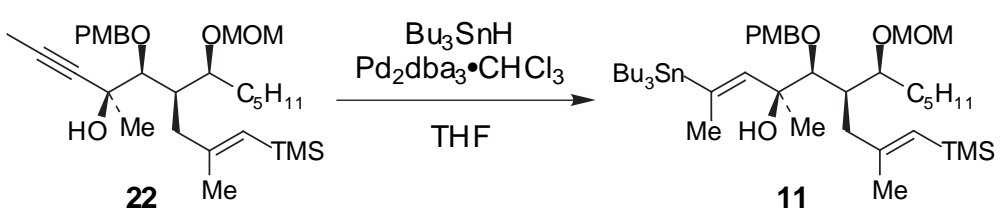

Synthesis of (1'S, $2 E, 4 S, 5 S, 6 R, 8 E)$ 2-[(Tributyl)stannyl]-4,8-dimethyl-5-pmethoxybenzyloxy-6-(1'-methoxymethoxy-hexyl)-9-(trimethylsilanyl)-nona-2,8-

dien-4-ol (11): To a solution of $22(1 \mathrm{~g}, 1.9 \mathrm{mmol})$ in THF $(3.8 \mathrm{~mL})$ at $23^{\circ} \mathrm{C}$ was added $\mathrm{Pd}_{2} \mathrm{dba}_{3} \cdot \mathrm{CHCl}_{3}$ (40 mg, 4\% mol.). $\mathrm{Bu}_{3} \mathrm{SnH}$ (10.1 mL, $38.5 \mathrm{mmol}, 20$ eq.) was then added dropwise over $1 \mathrm{~h} 30$ via a syringe pump addition. After $1 \mathrm{~h}$ at $23{ }^{\circ} \mathrm{C}$, the reaction mixture was concentrated. Purification of the crude material by flash chromatography on silica gel (hexanes / ethyl acetate $=100 / 0$ to $90 / 10$ ) afforded $0.764 \mathrm{~g}$ of $\mathbf{1 1}$ and $0.490 \mathrm{~g}$ of 22 . Resubjection of 22 to the same conditions gave after 2 recycles an additionnal $0.470 \mathrm{~g}$ of 11 (79\% after 2 recycles). ${ }^{1} \mathrm{H}$ NMR $\left(400 \mathrm{MHz} \mathrm{CDCl}_{3}\right) \delta 7.25(\mathrm{~d}, J=8.6 \mathrm{~Hz}, 2 \mathrm{H}), 6.87$ $(\mathrm{d}, J=8.6 \mathrm{~Hz}, 2 \mathrm{H}), 5.73\left(\mathrm{~d}, J=1.8 \mathrm{~Hz}, J_{S n-H}=79.7 \mathrm{~Hz}, 1 \mathrm{H}\right), 5.22(\mathrm{~s}, 1 \mathrm{H}), 4.65(\mathrm{~d}, J=$ $11.0 \mathrm{~Hz}, 1 \mathrm{H}), 4.64(\mathrm{~d}, J=6.6 \mathrm{~Hz}, 1 \mathrm{H}), 4.51(2 \mathrm{H}), 3.81(\mathrm{~s}, 1 \mathrm{H}), 3.80(\mathrm{~s}, 3 \mathrm{H}), 3.59(\mathrm{~m}$, 
1H), $3.50(\mathrm{~d}, J=4.8 \mathrm{~Hz}, 1 \mathrm{H}), 3.37(\mathrm{~s}, 3 \mathrm{H}), 2.65(\mathrm{dd}, J=13.8$ and $3.7 \mathrm{~Hz}, 1 \mathrm{H}), 2.35(\mathrm{~m}$, $1 \mathrm{H}), 2.16\left(\mathrm{~s}, J_{S n-H}=24.5 \mathrm{~Hz}, 3 \mathrm{H}\right), 2.02(\mathrm{dd}, J=13.8$ and $11.7 \mathrm{~Hz}, 1 \mathrm{H}), 1.77(\mathrm{~s}, 3 \mathrm{H}), 1.59-$ $1.39(11 \mathrm{H}), 1.34-1.23(12 \mathrm{H}), 0.93-0.80(18 \mathrm{H}), 0.09(\mathrm{~s}, 9 \mathrm{H}) ;{ }^{13} \mathrm{C} \mathrm{NMR}\left(100 \mathrm{MHz}, \mathrm{CDCl}_{3}\right)$ $\delta 158.9,153.7,144.5,140.7,131.0,128.5,126.2,113.6,95.3,84.1,78.7,78.0,74.0$, 55.8, 55.2, 41.0, 40.2, 31.7, 29.8, $29.2\left(J_{S n-C}=19.1 \mathrm{~Hz}\right), 27.4\left(J_{S n-C}=54.2 \mathrm{~Hz}\right), 26.5,22.7$, 20.9, 20.7, 14.1, 13.7, $9.2\left(J_{S n-C}=309.8 \mathrm{~Hz}\right),-0.1$; IR (thin film) $3414,2955,1614,1514$, 1250, 1090, 1040, 868, $840 \mathrm{~cm}^{-1}$; HRMS (electrospray) for $\mathrm{C}_{42} \mathrm{H}_{78} \mathrm{O}_{5} \mathrm{SiSnNa}[\mathrm{M}+\mathrm{Na}]^{+}$ calcd. 833.4538, found 833.4570; $[\alpha]_{\mathrm{D}}^{25}=-27.6\left(c 0.54, \mathrm{CHCl}_{3}\right)$.
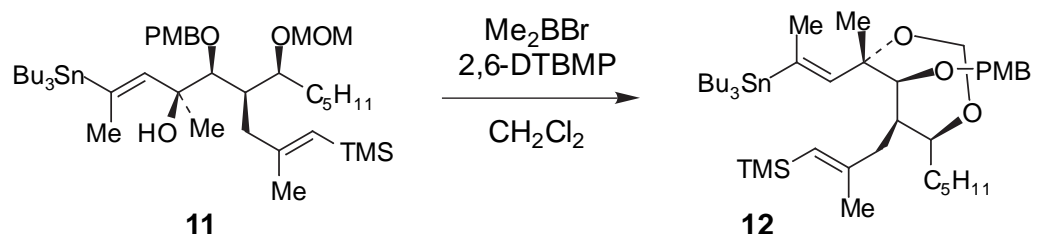

Synthesis of $\quad\left(1^{\prime} S, 2 E, 4 S, 5 S, 6 R\right)-2-[($ Tributyl)stannyl]-4-methyl-4,7-Omethylidene-5-p-methoxybenzyloxy-6-(E-2'-methyl-3'-trimethylsilanyl-2'-enyl $)$

dodec-2-ene (12): To a solution of $11(0.76 \mathrm{~g}, 0.94 \mathrm{mmol})$ and 2,6-di-t-butyl-4methylpyridine (2,6-DTBMP) (0.58 g, $2.80 \mathrm{mmol}, 3$ eq.) in $\mathrm{CH}_{2} \mathrm{Cl}_{2}(18 \mathrm{~mL})$ at $-78{ }^{\circ} \mathrm{C}$ was added $\mathrm{Me}_{2} \mathrm{BBr}(0.14 \mathrm{~mL}, 1.40 \mathrm{mmol}, 1.5$ eq. $)$ dropwise. After $30 \mathrm{~min}$. at $-60{ }^{\circ} \mathrm{C}$, an additionnal $0.15 \mathrm{~mL}$ of $\mathrm{Me}_{2} \mathrm{BBr}$ was added and the stirring was continued for $20 \mathrm{~min}$. The reaction mixture was then poured into an aqueous saturated solution of $\mathrm{NaHCO}_{3}$, and the aqueous layer was extracted with $\mathrm{CH}_{2} \mathrm{Cl}_{2}$. The combined organic layers were dried over $\mathrm{MgSO}_{4}$, filtered and concentrated. Purification of the crude material by flash chromatography on silica gel (hexanes / ethyl acetate $=100 / 0$ to $100 / 3$ ) afforded $0.674 \mathrm{~g}$ (92\%) of 12 as a colorless oil. ${ }^{1} \mathrm{H}$ NMR $\left(500 \mathrm{MHz}, \mathrm{CDCl}_{3}\right) \delta 7.24(\mathrm{~d}, J=8.7 \mathrm{~Hz}, 2 \mathrm{H})$, $6.86(\mathrm{~d}, J=8.7 \mathrm{~Hz}, 2 \mathrm{H}), 5.81\left(\mathrm{~d}, J=1.7 \mathrm{~Hz}, J_{S n-H}=77.4 \mathrm{~Hz}, 1 \mathrm{H}\right), 5.33(\mathrm{~s}, 1 \mathrm{H}), 4.92(\mathrm{~d}, J$ $=6.5 \mathrm{~Hz}, 1 \mathrm{H}), 4.87(\mathrm{~d}, J=6.5 \mathrm{~Hz}, 1 \mathrm{H}), 4.46(\mathrm{~d}, J=11.3 \mathrm{~Hz}, 1 \mathrm{H}), 4.43(\mathrm{~d}, J=11.3 \mathrm{~Hz}$, 
1H), $4.06(\mathrm{~m}, 1 \mathrm{H}), 3.81(\mathrm{~s}, 3 \mathrm{H}), 3.41(\mathrm{~d}, J=3.9 \mathrm{~Hz}, 1 \mathrm{H}), 2.39(\mathrm{dd}, J=13.9$ and $9.0 \mathrm{~Hz}$, 1H), $2.32(\mathrm{dd}, J=13.9$ and $5.9 \mathrm{~Hz}, 1 \mathrm{H}), 2.17(\mathrm{~m}, 1 \mathrm{H}), 2.00\left(\mathrm{~d}, J=2.0 \mathrm{~Hz}, J_{S n-H}=49 \mathrm{~Hz}\right.$, $3 \mathrm{H}), 1.75(\mathrm{~s}, 3 \mathrm{H}), 1.65-1.24(23 \mathrm{H}), 0.96-0.79(18 \mathrm{H}), 0.12(\mathrm{~s}, 9 \mathrm{H}) ;{ }^{13} \mathrm{C} \mathrm{NMR}(125 \mathrm{MHz}$, $\left.\mathrm{CDCl}_{3}\right) \delta 159.0,153.5,141.8,140.8,1309.9,128.9,126.4,113.6,89.3,85.5,83.7,75.0$ 72.9, 55.2, 45.0, 41.1, 32.4, 31.8, $29.2\left(J_{S n-C}=19.7 \mathrm{~Hz}\right), 27.4\left(J_{S n-C}=54.0 \mathrm{~Hz}\right), 26.3,25.0$, 22.6, 21.2, 20.8, 14.1, 13.7, $9.3\left(J_{S n-C}=318.6 \mathrm{~Hz}\right), 0.0$; IR (thin film) 2926, 1614, 1514, 1464, 1248, 1040, 865, $840 \mathrm{~cm}^{-1}$; HRMS (electrospray) for $\mathrm{C}_{41} \mathrm{H}_{74} \mathrm{O}_{5} \mathrm{SiSnNa}[\mathrm{M}+\mathrm{Na}]^{+}$ calcd. 801.4276, found 801.4299; $[\alpha]_{\mathrm{D}}^{25}=-39.5\left(c 0.20, \mathrm{CHCl}_{3}\right)$.
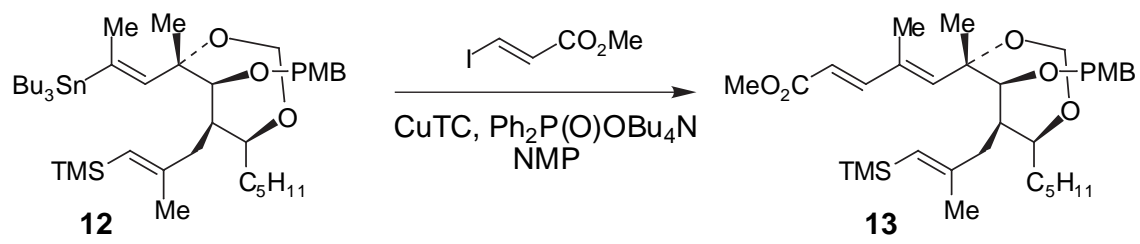

Synthesis of Methyl-(2E,4E,6S,7S,8R,9S)-4,6-dimethyl-6,9-O-methylidene-8( $E$-2'-methyl-3'-trimethylsilanyl-2'-enyl)-7-p-methoxybenzyloxy-tetradeca-2,4-

dienoate (13): To a solution of $12(70 \mathrm{mg}, 0.09 \mathrm{mmol})$,tetrabutyl ammonium diphenylphosphinate (45.5 mg, $0.1 \mathrm{mmol}, 1.1$ eq.) and CuTC (34 mg, $0.18 \mathrm{mmol}, 2$ eq.) in NMP $(0.5 \mathrm{~mL})$ at $23{ }^{\circ} \mathrm{C}$ was added a solution of $(E)$-ethyl $\beta$-iodoacrylate $(34 \mathrm{mg}, 0.16$ mmol, 1.8 eq. $)$ in NMP (0.3 mL) over 45 min via a syringe pump addition. After the addition was complete, the reaction mixture was diluted with $\mathrm{Et}_{2} \mathrm{O}$ and filtered through neutral alumina. The filtrate was successively washed with water $(6 \mathrm{~mL})$ and brine. The organic layer was dried over $\mathrm{MgSO}_{4}$, filtered and concentrated. Purification of the crude material by preparative thin layer chromatography (hexanes $/$ ethyl acetate $=90 / 10$ ) gave $47.4 \mathrm{mg}(92 \%)$ of 13 as a colorless oil. ${ }^{1} \mathrm{H}$ NMR $\left(500 \mathrm{MHz}, \mathrm{CDCl}_{3}\right) \delta 7.23(\mathrm{~d}, J=8.7 \mathrm{~Hz}$, 2H), $7.19(\mathrm{~d}, J=15.8 \mathrm{~Hz}, 1 \mathrm{H}), 6.90(\mathrm{~d}, J=8.7 \mathrm{~Hz}, 2 \mathrm{H}), 5.82(\mathrm{~d}, J=15.8 \mathrm{~Hz}, 1 \mathrm{H}), 5.74$ $(\mathrm{s}, 1 \mathrm{H}), 5.33(\mathrm{~s}, 1 \mathrm{H}), 4.94(\mathrm{~d}, J=5.9 \mathrm{~Hz}, 1 \mathrm{H}), 4.81(\mathrm{~d}, J=5.9 \mathrm{~Hz}, 1 \mathrm{H}), 4.56(\mathrm{~d}, J=11.7$ 
$\mathrm{Hz}, 1 \mathrm{H}), 4.29(\mathrm{~d}, J=11.7 \mathrm{~Hz}, 1 \mathrm{H}), 4.10(\mathrm{~m}, 1 \mathrm{H}), 3.85(\mathrm{~s}, 3 \mathrm{H}), 3.77(\mathrm{~s}, 3 \mathrm{H}), 3.34$ (d, $J=$ $3.7 \mathrm{~Hz}, 1 \mathrm{H}), 2.40-2.32(2 \mathrm{H}), 2.17(\mathrm{~m}, 1 \mathrm{H}), 1.86(\mathrm{~s}, 3 \mathrm{H}), 1.85(\mathrm{~s}, 3 \mathrm{H}), 1.68(\mathrm{~m}, 1 \mathrm{H}), 1.51$ $(\mathrm{s}, 3 \mathrm{H}), 1.48-1.27(7 \mathrm{H}), 0.91(\mathrm{t}, J=6.8 \mathrm{~Hz}, 3 \mathrm{H}), 0.15(\mathrm{~s}, 9 \mathrm{H}) ;{ }^{13} \mathrm{C} \mathrm{NMR}(125 \mathrm{MHz}$, $\left.\mathrm{CDCl}_{3}\right) \delta 167.8,159.4,153.1,150.8,144.9,133.0,130.1,129.8,126.7,115.6,113.8$ $89.4,83.5,82.3,74.4,71.8,55.3,51.4,44.4,40.3,32.2,21.7,26.2,22.8,22.6,21.4,14.0$, 12.8, 0.0; IR (thin film) 2953, 1721, 1619, 1515, 1301, 1249, 1060, 1040, $840 \mathrm{~cm}^{-1}$; HRMS (electrospray) for $\mathrm{C}_{33} \mathrm{H}_{52} \mathrm{O}_{5} \mathrm{SiNa}[\mathrm{M}+\mathrm{Na}]^{+}$calcd. 595.3431, found 595.3435; $[\alpha]_{\mathrm{D}}^{25}=-63.8\left(c 0.50, \mathrm{CHCl}_{3}\right)$.

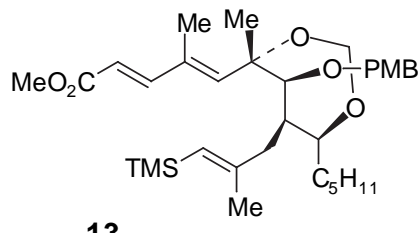

13

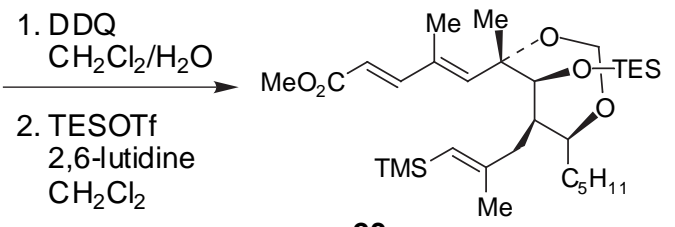

23

Synthesis of Methyl-(2E,4E,6S,7S,8R,9S)-4,6-dimethyl-6,9-O-methylidene-8(E-2'-methyl-3' -trimethylsilanyl-2' -enyl)-7-[(triethylsilyl)oxy]-tetradeca-2,4-dienoate (23): To a solution of $13(29 \mathrm{mg}, 0.05 \mathrm{mmol})$ in $\mathrm{CH}_{2} \mathrm{Cl}_{2} / \mathrm{H}_{2} \mathrm{O}(1 \mathrm{~mL} / 0.1 \mathrm{~mL})$ at $0{ }^{\circ} \mathrm{C}$ was added DDQ (17.2 mg, $0.08 \mathrm{mmol}, 1.5$ eq.). After $1 \mathrm{~h}$ at $0{ }^{\circ} \mathrm{C}$ and $1 \mathrm{~h}$ at $23{ }^{\circ} \mathrm{C}$, the reaction mixture was diluted with an aqueous saturated solution of $\mathrm{NaHCO}_{3}$. The aqueous layer was extracted with $\mathrm{CH}_{2} \mathrm{Cl}_{2}$, the combined organic layers were washed with brine, dried over $\mathrm{MgSO}_{4}$, filtered and concentrated. The crude material was dissolved in $\mathrm{CH}_{2} \mathrm{Cl}_{2}(0.5$ $\mathrm{mL})$ and cooled to $0{ }^{\circ} \mathrm{C}$. 2,6-Lutidine $(18 \mu \mathrm{L}, 0.15 \mathrm{mmol}, 3$ eq. $)$ was then added, followed by TESOTf $\left(23 \mu \mathrm{L}, 0.10 \mathrm{mmol}, 2\right.$ eq.). After $30 \mathrm{~min}$ at $0{ }^{\circ} \mathrm{C}$, the reaction mixture was quenched by addition of an aqueous saturated solution of $\mathrm{NaHCO}_{3}$. The aqueous layer was extracted with $\mathrm{CH}_{2} \mathrm{Cl}_{2}$, the combined organic layers were washed with brine, dried over $\mathrm{MgSO}_{4}$, filtered and concentrated. Filtration through a pad of silica gel (hexanes / ethyl acetate $=80 / 20)$ followed by HPLC (hexanes $/$ ethyl acetate $=85 / 15$ ) afforded 22.2 
mg (77\% from 13) of 23 as a colorless oil. ${ }^{1} \mathrm{H}$ NMR $\left(500 \mathrm{MHz}, \mathrm{CDCl}_{3}\right) \delta 7.33(\mathrm{~d}, J=$ $15.5 \mathrm{~Hz}, 1 \mathrm{H}), 6.20(\mathrm{~s}, 1 \mathrm{H}), 5.87(\mathrm{~d}, J=15.5 \mathrm{~Hz}, 1 \mathrm{H}), 5.31(\mathrm{~s}, 1 \mathrm{H}), 4.89$ (d, $J=6.6 \mathrm{~Hz}$, $1 \mathrm{H}), 4.74(\mathrm{~d}, J=6.6 \mathrm{~Hz}, 1 \mathrm{H}), 4.02(\mathrm{~m}, 1 \mathrm{H}), 3.82(\mathrm{~d}, J=4.2 \mathrm{~Hz}, 1 \mathrm{H}), 3.77(\mathrm{~s}, 3 \mathrm{H}), 2.38$ $(\mathrm{dd}, J=14.9$ and $8.6 \mathrm{~Hz}, 1 \mathrm{H}), 2.30(\mathrm{dd}, J=14.9$ and $5.6 \mathrm{~Hz}, 1 \mathrm{H}), 1 . .98(\mathrm{~m}, 1 \mathrm{H}), 1.93(\mathrm{~s}$, $3 \mathrm{H}), 1.82(\mathrm{~s}, 3 \mathrm{H}), 1.65(\mathrm{~m}, 1 \mathrm{H}), 1.49(\mathrm{~s}, 3 \mathrm{H}), 1.45-1.41(2 \mathrm{H}), 1.36-1.24(5 \mathrm{H}), 0.97(\mathrm{t}, J=$ $8.0 \mathrm{~Hz}, 9 \mathrm{H}), 0.89(\mathrm{t}, J=7.0 \mathrm{~Hz}, 3 \mathrm{H}), 0.62(\mathrm{q}, J=8.0 \mathrm{~Hz}, 6 \mathrm{H}), 0.12(\mathrm{~s}, 9 \mathrm{H}) ;{ }^{13} \mathrm{C} \mathrm{NMR}$ $\left(125 \mathrm{MHz}, \mathrm{CDCl}_{3}\right) \delta 167.9,153.4,150.8,144.0,133.2,125.8,115.8,89.9,83.1,78.8$ $75.0,51.5,48.6,40.2,32.5,31.8,26.3,25.3,22.6,21.8,14.0,12.9,7.0,5.2,0.0$; IR (thin film) $2955,1724,1622,1300,1250,1170,1070,1020,840 \mathrm{~cm}^{-1}$; HRMS (electrospray) for $\mathrm{C}_{31} \mathrm{H}_{58} \mathrm{O}_{5} \mathrm{Si}_{2} \mathrm{Na}[\mathrm{M}+\mathrm{Na}]^{+}$calcd. 589.3721, found 589.3727; $[\alpha]_{\mathrm{D}}^{25}=-20.4$ (c 0.93, $\left.\mathrm{CHCl}_{3}\right)$.

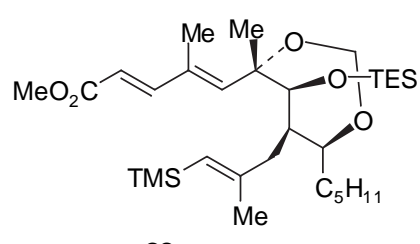

23

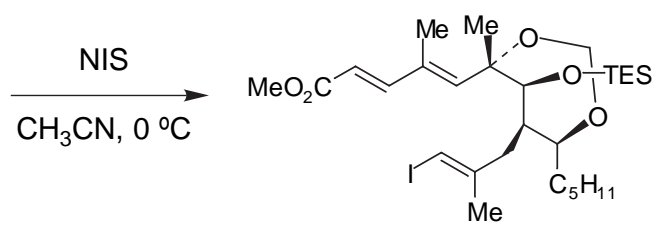

5

Synthesis of Methyl-(2E,4E,6S,7S,8R,9S)-4,6-dimethyl-6,9-O-methylidene-8(E-2'-methyl-3'-iodoprop-2' -enyl)-7-[(triethylsilyl)oxy]-tetradeca-2,4-dienoate (6): To a solution of $23(13.9 \mathrm{mg}, 0.024 \mathrm{mmol})$ in $\mathrm{CH}_{3} \mathrm{CN}(0.5 \mathrm{~mL})$ at $0{ }^{\circ} \mathrm{C}$ was added NIS (6.6 $\mathrm{mg}, 0.03 \mathrm{mmol}, 1.2$ eq.) in one portion. After $2 \mathrm{~h}$ at $0{ }^{\circ} \mathrm{C}$, the reaction mixture was quenched by the addition of an aqueous saturated solution of $\mathrm{Na}_{2} \mathrm{~S}_{2} \mathrm{O}_{3}(3 \mathrm{~mL})$. The aqueous layer was extracted with $\mathrm{CH}_{2} \mathrm{Cl}_{2}$, the combined organic layers were washed with brine, dried over $\mathrm{MgSO}_{4}$, filtered and concentrated. Purification of the crude material by preparative thin layer chromatography (hexanes $/$ ethyl acetate $=90 / 10$ ) gave $13.3 \mathrm{mg}$ $(87 \%)$ of 6 as a colorless oil. ${ }^{1} \mathrm{H}$ NMR $\left(500 \mathrm{MHz}, \mathrm{CDCl}_{3}\right) \delta 7.32(\mathrm{~d}, J=15.7 \mathrm{~Hz}, 1 \mathrm{H})$, 
$6.25(\mathrm{~s}, 1 \mathrm{H}), 6.00(\mathrm{~s}, 1 \mathrm{H}), 5.87(\mathrm{~d}, J=15.7 \mathrm{~Hz}, 1 \mathrm{H}), 4.87\left(\mathrm{~A}\right.$ of $\left.\mathrm{AB}, J_{A B}=6.6 \mathrm{~Hz}, 1 \mathrm{H}\right)$, $4.72\left(\mathrm{~B}\right.$ of $\left.\mathrm{AB}, J_{A B}=6.6 \mathrm{~Hz}, 1 \mathrm{H}\right), 3.95(\mathrm{~m}, 1 \mathrm{H}), 3.76(\mathrm{~s}, 3 \mathrm{H}), 3.73(\mathrm{~d}, J=3.6 \mathrm{~Hz}, 1 \mathrm{H})$, $2.52\left(\mathrm{~A}\right.$ of $\left.\mathrm{ABX}, J_{A B}=14.6 \mathrm{~Hz}, J_{A X}=8.6 \mathrm{~Hz}, 1 \mathrm{H}\right), 2.42\left(\mathrm{~B}\right.$ of $\mathrm{ABX}, J_{A B}=14.6 \mathrm{~Hz}, J_{B X}=$ $5.2 \mathrm{~Hz}, 1 \mathrm{H}), 1.91(\mathrm{~s}, 3 \mathrm{H}), 1.88(\mathrm{~m}, 1 \mathrm{H}), 1.86(\mathrm{~s}, 3 \mathrm{H}), 1.64(\mathrm{~m}, 1 \mathrm{H}), 1.48$ (s, $3 \mathrm{H}), 1.42$ $(\mathrm{m}, 1 \mathrm{H}), 1.32-1.25(\mathrm{~m}, 6 \mathrm{H}), 0.95(\mathrm{t}, J=8.0 \mathrm{~Hz}, 9 \mathrm{H}), 0.89(\mathrm{t}, J=6.9 \mathrm{~Hz}, 3 \mathrm{H}), 0.59(\mathrm{q}, J$ $=8.0 \mathrm{~Hz}, 6 \mathrm{H}) ;{ }^{13} \mathrm{C} \mathrm{NMR}\left(125 \mathrm{MHz}, \mathrm{CDCl}_{3}\right) \delta 167.8,150.5,146.5,142.6,133.9,116.1$, $90.0,83.2,78.9,76.6,74.9,51.5,49.0,37.9,32.7,31.7,26.2,26.1,24.0,22.6,14.0,13.0$, 7.0 (3), 5.2 (3); IR (thin film) 3059, 2954, 2932, 2876, 1722, 1623, 1458, 1433, 1377 , 1298, 1270, 1170, 1121, 1074, 1019, 841, $726 \mathrm{~cm}^{-1}$; HRMS (FAB) for $\mathrm{C}_{28} \mathrm{H}_{49} \mathrm{IO}_{5} \mathrm{SiNa}$ $[\mathrm{M}+\mathrm{Na}]^{+}$calcd. 643.2292, found 643.2295; $[\alpha]_{\mathrm{D}}^{24}-9.2\left(c=0.34, \mathrm{CHCl}_{3}\right)$.
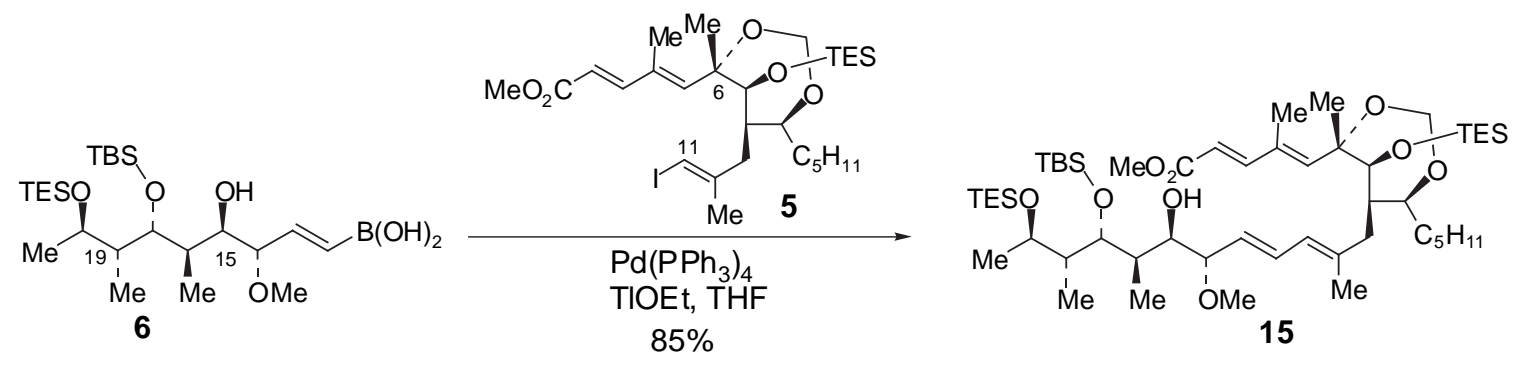

Synthesis of Methyl-(6S,7S,8R,9S)-4,6-dimethyl-6,9-O-methylidene-8-\{(1'E$\left.3^{\prime} E, 5^{\prime} S, 6^{\prime} R, 7^{\prime} R, 8^{\prime} R, 9^{\prime} R, 10^{\prime} R\right)-8^{\prime}$-[(tert-butyldimethylsilyl)oxy]-10'-[(triethylsilyl)oxy)]-6'-(hydroxy)-[1',7',9'-trimethyl]-5' -(methoxy)-undeca-1',3'-diene $\}-7$ -

[(triethylsilyl)oxy]-icoso-2,4,10,12-tetraenoate (15): The boronic acid 6 (153 $\mathrm{mg}, 0.30$ mmol) and vinyl iodide 5 (157 $\mathrm{mg}, 0.25 \mathrm{mmol})$ were combined in $4 \mathrm{~mL}$ of degassed $\mathrm{THF} / \mathrm{H}_{2} \mathrm{O}(4: 1 \mathrm{v}: \mathrm{v})$ and the solution was further degassed via one freeze-pump-thaw cycle. The reaction was treated sequentially with $\mathrm{Pd}\left(\mathrm{Ph}_{3} \mathrm{P}\right)_{4}(29 \mathrm{mg}, 0.025 \mathrm{mmol})$, and $\mathrm{Tl}(\mathrm{OEt})(33 \mu \mathrm{L}, 0.45 \mathrm{mmol})$ and the mixture stirred at $23{ }^{\circ} \mathrm{C}$. After $75 \mathrm{~min}$, additional $\mathrm{Pd}\left(\mathrm{Ph}_{3} \mathrm{P}\right)_{4}(10 \mathrm{mg}, 0.009 \mathrm{mmol})$, and $\mathrm{Tl}(\mathrm{OEt})(10 \mu \mathrm{L}, 0.13 \mathrm{mmol})$ were added and the 
reaction stirred for an additional $15 \mathrm{~min}$, at which time TLC analysis indicated the reaction was complete. The reaction mixture was diluted with $\mathrm{Et}_{2} \mathrm{O} /$ hexanes $(1: 1)$, filtered through Celite to remove salts, and the residue concentrated. Purification of this crude product by column chromatography $\left(15 \mathrm{~g} \mathrm{SiO}_{2}, 5 \%-10 \%\right.$ EtOAc/hexanes $)$ provided $206 \mathrm{mg}(85 \%)$ of $15 .{ }^{1} \mathrm{H} \mathrm{NMR}\left(\mathrm{CDCl}_{3}, 500 \mathrm{MHz}\right) \delta 7.26(\mathrm{~d}, J=14.3 \mathrm{~Hz}, 1 \mathrm{H})$, $6.45(\mathrm{dd}, J=15.1,10.7 \mathrm{~Hz}, 1 \mathrm{H}), 6.21(\mathrm{~s}, 1 \mathrm{H}), 5.99(\mathrm{~d}, J=10.7 \mathrm{~Hz}, 1 \mathrm{H}), 5.83(\mathrm{~d}, J=$ $15.6 \mathrm{~Hz}, 1 \mathrm{H}), 5.56$ (dd, $J=15.1,8.1 \mathrm{~Hz}, 1 \mathrm{H}), 4.91(\mathrm{~A}$ of AB, $J=6.5 \mathrm{~Hz}, 1 \mathrm{H}$ ), 4.76 (B of AB, $J=6.5 \mathrm{~Hz}, 1 \mathrm{H}), 4.00(\mathrm{dd}, J=9.2,2.00 \mathrm{~Hz}, 1 \mathrm{H}), 3.92(\mathrm{~d}, J=8.1 \mathrm{~Hz}, 1 \mathrm{H}), 3.82$ to 3.72 (m, 3H), 3.75 (s, 3H), 3.49, (app. t, $J=8.1 \mathrm{~Hz}, 1 \mathrm{H}), 3.35$ (s, 1H), 3.25 (s, 3H), 2.34 (dd, $J=14.4,8.8 \mathrm{~Hz}, 1 \mathrm{H}), 2.24$ (dd, $J=14.4,4.6 \mathrm{~Hz}, 1 \mathrm{H}), 2.01$ to $1.95(\mathrm{~m}, 1 \mathrm{H}), 1.93$ to $1.80(\mathrm{~m}, 2 \mathrm{H}), 1.91(\mathrm{~s}, 3 \mathrm{H}), 1.79(\mathrm{~s}, 3 \mathrm{H}), 1.70$ to $1.60(\mathrm{~m}, 1 \mathrm{H}), 1.51(\mathrm{~s}, 3 \mathrm{H}), 1.48$ to 1.38 (m, 1H), 1.38 to $1.20(\mathrm{~m}, 6 \mathrm{H}), 1.05(\mathrm{~d}, J=6.1 \mathrm{~Hz}, 3 \mathrm{H}), 1.01(\mathrm{~d}, J=7.1 \mathrm{~Hz}, 3 \mathrm{H}), 0.99$ to $0.85(\mathrm{~m}, 18 \mathrm{H}), 0.89$ (s, 12H), 0.88 (t, $J=3.2 \mathrm{~Hz}, 3 \mathrm{H}), 0.64$ to $0.55(\mathrm{~m}, 12 \mathrm{H}), 0.10$ (s, 3H), 0.08 (s, 3H); ${ }^{13} \mathrm{C} \mathrm{NMR}\left(\mathrm{CDCl}_{3}, 125 \mathrm{MHz}\right) \quad \delta 167.9,150.7,143.7,137.1,133.4$, 130.6, 129.8, 126.7, 115.8, 89.9, 83.2, 82.3, 79.5, 78.8, 77.2, 74.9, 73.5, 70.1, 55.9, 51.5, 49.1, 44.3, 37.6, 37.6, 32.8, 31.7, 26.2, 25.5, 22.6, 19.9, 18.3, 16.9, 14.0, 12.9, 11.7, 11.6, 7.0, 6.9, 5.2, -3.5, -3.7; IR (neat) 2956, 2878, 1724, 1623, 1463, 1298, 1253, 1170, 1075, 1017, 837, 774, $742 \mathrm{~cm}^{-1}$; HRMS (FAB+) for $\mathrm{C}_{52} \mathrm{H}_{100} \mathrm{O}_{9} \mathrm{NaSi}_{3}[\mathrm{M}+\mathrm{Na}]^{+}$calcd. 975.6573, found 975.6527; $[\alpha]^{26}-21.2\left(c 0.92, \mathrm{CHCl}_{3}\right)$.
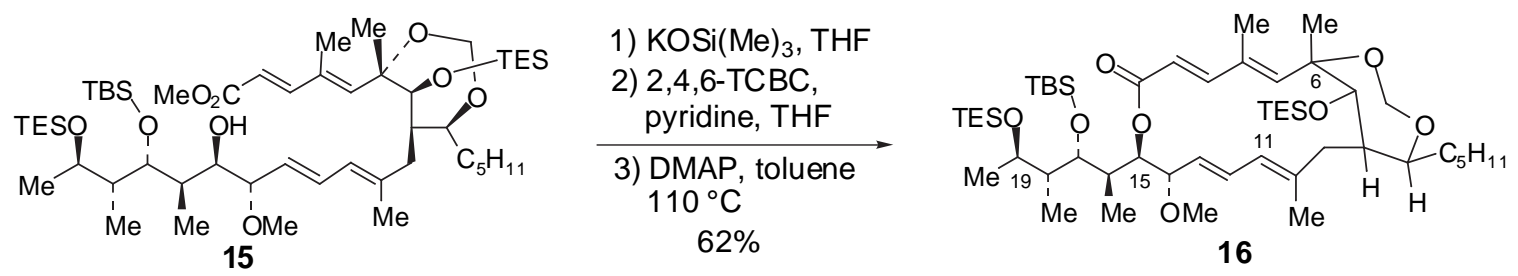
Synthes is of

$(1 S, 2 E, 4 E, 8 R, 9 S, 10 E, 12 E, 15 R, 16 S, 20 S)-8-$

$\left[\left(1^{\prime} R, 2^{\prime} R, 3^{\prime} S, 6^{\prime} R, 7^{\prime} S, 8^{\prime} R\right)-2^{\prime}, 7^{\prime}\right.$-[di(tert-Butyldimethylsilyl)oxy]-4'-[(triethylsilyl)oxy]$\left(1^{\prime}, 3^{\prime}\right.$,dimethyl)-pentyl]-20-[(triethylsilyl)oxy]-9-methoxy-1,3,13-trimethyl-16-pentyl7,17,19-trioxabicyclo[13.4.1] eicosa-2,4,10,12-tetraene-6-one (16): To the ester 15 (130 $\mathrm{mg}, 0.14 \mathrm{mmol})$ in $5.5 \mathrm{~mL}$ of $\mathrm{Et}_{2} \mathrm{O} / \mathrm{THF}(10: 1)$ was added $\mathrm{KOSi}(\mathrm{Me})_{3}(39 \mathrm{mg}, 0.30$ $\mathrm{mmol})$, and the mixture stirred for $24 \mathrm{~h}$. The reaction was diluted with EtOAc $(25 \mathrm{~mL})$ and $\mathrm{H}_{2} \mathrm{O}(5 \mathrm{~mL})$, and the biphasic mixture stirred vigorously for $5 \mathrm{~min}$. The organic layer was separated and the aqueous was made acidic by the addition of $1 \mathrm{~N} \mathrm{HCl}(500 \mu \mathrm{L})$, and further extracted with EtOAc. The combined organic solution was washed with brine, dried over $\mathrm{Na}_{2} \mathrm{SO}_{4}$, filtered and concentrated in vacuo to provide $126 \mathrm{mg}$ of the crude acid which was used without further purification.

The crude acid (126 mg, $0.14 \mathrm{mmol}$ ) was azeotropically dried by concentration from benzene $(3 \mathrm{x})$ on a rotary evaporator. The residue was dissolved in $3 \mathrm{~mL}$ of THF and treated sequentially with $i$-Pr ${ }_{2} \mathrm{NEt}(356 \mu \mathrm{L}, 2.1 \mathrm{mmol})$ and 2,4,6trichlorobenzoylchloride (TCBC) $174 \mu \mathrm{L}, 1.1 \mathrm{mmol}$ ), and the reaction stirred at $23{ }^{\circ} \mathrm{C}$ for $2 \mathrm{~h}$. The reaction mixture was concentrated in vacuo and the residue was dissolved in 1.5 $\mathrm{mL}$ of toluene, and added to a solution of 4-dmimethylaminopyridine (DMAP) (166 mg $1.36 \mathrm{mmol})$, followed by $2 \mathrm{~mL}$ of toluene rinse. The reaction was heated to reflux for 13 h, cooled to $23{ }^{\circ} \mathrm{C}$ and filtered. The filtrate was diluted with $\mathrm{Et}_{2} \mathrm{O}$ and washed with $\mathrm{H}_{2} \mathrm{O}$, brine, dried over $\mathrm{Na}_{2} \mathrm{SO}_{4}$, filtered and concentrated in vacuo. The residue was purified by column chromatography (10 g of Davisil, 2\% - 5\% EtOAc/hexanes) followed by HPLC (21 mm column, 5\% EtOAc/hexanes) to provide $78 \mathrm{mg}(62 \%)$ of 16. ${ }^{1} \mathrm{H} \mathrm{NMR}\left(\mathrm{CDCl}_{3}\right.$, $500 \mathrm{MHz}) \delta 7.10(\mathrm{~d}, J=15.6 \mathrm{~Hz}, 1 \mathrm{H}), 6.49(\mathrm{dd}, J=14.9,10.7 \mathrm{~Hz}, 1 \mathrm{H}), 5.8(\mathrm{~d}, J=10.7$ $\mathrm{Hz}, 1 \mathrm{H}), 5.70(\mathrm{~d}, J=15.8 \mathrm{~Hz}, 1 \mathrm{H}) 5.65(\mathrm{~s}, 1 \mathrm{H}), 5.22(\mathrm{dd}, J=14.9,8.6 \mathrm{~Hz}, 1 \mathrm{H}), 5.02$ (dd, $J=7.6,4.6 \mathrm{~Hz}, 1 \mathrm{H}), 4.76(\mathrm{~d}, J=8.8 \mathrm{~Hz}, 1 \mathrm{H}), 4.68(\mathrm{~d}, J=8.8 \mathrm{~Hz}, 1 \mathrm{H}), 3.96$ (app. $\mathrm{t}, J=$ $3.4 \mathrm{~Hz}, 1 \mathrm{H}), 3.88$ (app. t, $J=8.1 \mathrm{~Hz}, 1 \mathrm{H}), 3.83(\operatorname{app~t}, J=6.4 \mathrm{~Hz}, 1 \mathrm{H}), 3.79(\mathrm{~d}, J=7.3$ $\mathrm{Hz}, 1 \mathrm{H}), 3.64$ to $3.58(\mathrm{~m}, 1 \mathrm{H}), 3.24(\mathrm{~s}, 3 \mathrm{H}), 2.67$ (dd, $J=7.3,6.9 \mathrm{~Hz}, 1 \mathrm{H}), 2.09$ to 2.02 
(m, 1H), $1.99(\mathrm{dd}, J=9.0,4.9 \mathrm{~Hz}, 1 \mathrm{H}), 1.96(\mathrm{~s}, 3 \mathrm{H}), 1,95(\mathrm{~d}, J=8.3,1 \mathrm{H}), 1.92$ to 1.85 $(\mathrm{m}, 1 \mathrm{H}), 1.86(\mathrm{~s}, 3 \mathrm{H}), 1.65$ to $1.55(\mathrm{~m}, 1 \mathrm{H}), 1.40$ to $1.20(\mathrm{~m}, 7 \mathrm{H}), 1.39(\mathrm{~s}, 3 \mathrm{H}), 1.10(\mathrm{~d}, J$ $=6.4 \mathrm{~Hz}, 3 \mathrm{H}), 1.02(\mathrm{t}, J=8.1 \mathrm{~Hz}, 12 \mathrm{H}), 0.95(\mathrm{t}, J=7.8 \mathrm{~Hz}, 9 \mathrm{H}), 0.88(\mathrm{t}, J=3.4 \mathrm{~Hz}$, 3H), $0.88(\mathrm{~s}, 12 \mathrm{H}), 0.81$ to $0.69(\mathrm{~m}, 6 \mathrm{H}), 0.61$ (q, $J=7.8 \mathrm{~Hz}, 6 \mathrm{H}), 0.09$ (s, 3H), 0.07 (s, $3 \mathrm{H}) ;{ }^{13} \mathrm{C} \mathrm{NMR}\left(\mathrm{CDCl}_{3}, 125 \mathrm{MHz}\right) \quad \delta 166.7,150.8,142.2,141.2,134.4,131.6,128.2$, $125.1,116.5,86.4,85.4,81.2,78.6,75.5,74.9,73.2,70.3,55.7,47.4,43.1,42.6,34.2$, $31.9,30.2,26.1,25.9,25.5,22.6,20.1,20.0,18.5,14.1,12.0,11.5,10.2,7.3,7.1,5.7,5.3$, $-3.6,-4.1$; IR (neat) 2957, 2878, 1713, 1461, 1373, 1253, 1089, 1018, 967, 869, 835, 773, 736, $674 \mathrm{~cm}^{-1}$; HRMS (FAB+) for $\mathrm{C}_{51} \mathrm{H}_{58} \mathrm{O}_{4} \mathrm{NaSi}_{3}[\mathrm{M}+\mathrm{Na}]^{+}$calcd. 943.6310, found 943.6318; $[\alpha]^{26}-2.3\left(c 0.78, \mathrm{CHCl}_{3}\right)$.

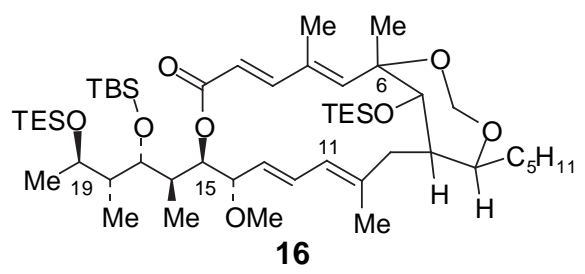

Synthesis

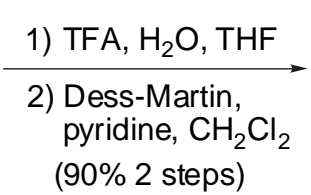

(90\% 2 steps)

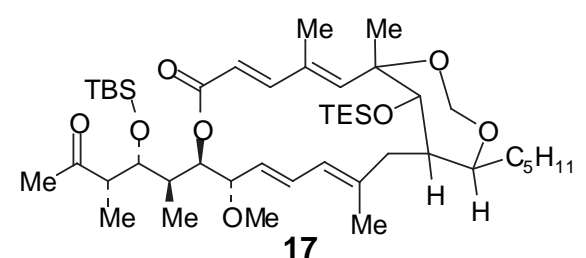

$(1 S, 2 E, 4 E, 8 R, 9 S, 10 E, 12 E, 15 R, 16 S, 20 S)-8-$

$\left[\left(1^{\prime} R, 2^{\prime} R, 3^{\prime} S, 6^{\prime} R, 7^{\prime} S, 8^{\prime} R\right)-2^{\prime}, 7^{\prime}\right.$-di(tert-Butyldimethylsilyl)oxy-(1',3'-dimethyl)-pent-4' one]-20-[(trimethylsilyl)oxy]-9-methoxy-1,3,13-trimethyl-16-pentyl-7,17,19trioxabicyclo[13.4.1] eicosa-2,4,10,12-tetraene-6-one (17). A solution of the macrolactone $16(78 \mathrm{mg}, 0.085 \mathrm{mmol})$ in $\mathrm{THF}(2.5 \mathrm{~mL})$ was cooled to $0{ }^{\circ} \mathrm{C}$ and a solution of TFA $\left(212 \mu \mathrm{L}, 4.0 \mathrm{M}\right.$ in $\left.\mathrm{H}_{2} \mathrm{O}\right)$ was added. The reaction was stirred for $150 \mathrm{~min}$ until complete by TLC analysis (20\% ethyl acetate/hexanes). The reaction was diluted with $15 \mathrm{~mL}$ of $\mathrm{Et}_{2} \mathrm{O}$ and washed with $\mathrm{NaHCO}_{3}(3 \times 2 \mathrm{~mL})$, dried over $\mathrm{MgSO}_{4}$, filtered, and concentrated on the rotary evaporator followed by high vacuum to give $72 \mathrm{mg}$ 
(>99\%) of crude alcohol which was $>95 \%$ pure by ${ }^{1} \mathrm{H}-\mathrm{NMR}$ and was used without purification.

A solution of the crude alcohol (72 $\mathrm{mg}$ crude, $0.085 \mathrm{mmol})$ in $\mathrm{CH}_{2} \mathrm{Cl}_{2}(3.0 \mathrm{~mL})$ at $0{ }^{\circ} \mathrm{C}$ was treated with pyridine ( $34 \mu \mathrm{L}, 0.42 \mathrm{mmol}$ ), followed by Dess-Martin periodinane (72 $\mathrm{mg}, 0.17 \mathrm{mmol})$. The reaction stirred at $0{ }^{\circ} \mathrm{C}$ for $2 \mathrm{~h}$. TLC analysis $(20 \%$ ethyl acetate/hexanes) at this point showed the reaction to be approximately $70 \%$ complete. Additional pyridine $(68 \mu \mathrm{L}, 0.84 \mathrm{mmol})$ and Dess-Martin periodinane $(72 \mathrm{mg}, 0.17$ mmol) were added and the reaction stirred until complete by TLC analysis $(4 \mathrm{~h})$. The reaction mixture was diluted with $\mathrm{Et}_{2} \mathrm{O}(20 \mathrm{~mL})$ and washed $(3 \times 2 \mathrm{~mL})$ with a $1: 1(\mathrm{v}: \mathrm{v})$ mixture of satd. $\mathrm{Na}_{2} \mathrm{~S}_{2} \mathrm{O}_{3}$ and satd. $\mathrm{NaHCO}_{3}$, dried over $\mathrm{MgSO}_{4}$, filtered, and concentrated on the rotary evaporator. The crude residue was purified on a Davisil ${ }^{\circledR}$ column $(5.0 \mathrm{~g})$ eluted with a gradient of ethyl acetate/hexanes (5-20\%) to yield $61 \mathrm{mg}$ (90\% from macrolactone 16) of the methyl ketone 17: ${ }^{1} \mathrm{H}$ NMR $\left(\mathrm{CDCl}_{3}, 500 \mathrm{MHz}\right) \delta 7.13(\mathrm{~d}, J=$ $15.9 \mathrm{~Hz}, 1 \mathrm{H}), 6,48(\mathrm{dd}, J=15.1,11.0 \mathrm{~Hz}, 1 \mathrm{H}), 5.78(\mathrm{~d}, J=11.0 \mathrm{~Hz}, 1 \mathrm{H}), 5.72(\mathrm{~d}, J=$ $15.9 \mathrm{~Hz}, 1 \mathrm{H}), 5.66$ (s, 1H), $5.14(\mathrm{dd}, J=15.1,9.5 \mathrm{~Hz}, 1 \mathrm{H}), 4.91(\mathrm{dd}, J=7.8,5.6 \mathrm{~Hz}$, 1H), 4.77 (d, $J=8.5 \mathrm{~Hz}, 1 \mathrm{H}), 4.70$ (d, $J=8.5 \mathrm{~Hz}, 1 \mathrm{H}), 4.30$ (app. t, $J=3.7 \mathrm{~Hz}, 1 \mathrm{H}$ ), $3.80(\mathrm{~d}, J=7.3 \mathrm{~Hz}, 1 \mathrm{H}), 3.70$ (d, $J=8.8 \mathrm{~Hz}, 1 \mathrm{H}) 3.61$ (app. t, $J=7.6 \mathrm{~Hz}, 1 \mathrm{H}), 3.19$ (s, $3 \mathrm{H}), 2.95$ (ddd, $J=11.0,10.3,3.2 \mathrm{~Hz}, 1 \mathrm{H}), 2,75$ to $2.64(\mathrm{~m}, 1 \mathrm{H}), 2.18,(\mathrm{~s}, 3 \mathrm{H}), 2.04$ to $1.90(\mathrm{~m}, 2 \mathrm{H}), 1.98(\mathrm{~s}, 3 \mathrm{H}), 1.88(\mathrm{~s}, 3 \mathrm{H}), 1.64$ to $1.56(\mathrm{~m}, 1 \mathrm{H}), 1.38(\mathrm{~s}, 3 \mathrm{H}), 1.38$ to 1.22 $(\mathrm{m}, 8 \mathrm{H}), 1.13(\mathrm{~d}, J=7.1 \mathrm{~Hz}, 3 \mathrm{H}), 1.02(\mathrm{~d}, J=7.8 \mathrm{~Hz}, 3 \mathrm{H}), 1.00(\mathrm{t}, J=5.6 \mathrm{~Hz}, 9 \mathrm{H}), 0.89$ (t, $J=6.8 \mathrm{~Hz}, 3 \mathrm{H}), 0.88$ (s, 9H), 0.81 to $0.70(\mathrm{~m}, 6 \mathrm{H}), 0.15(\mathrm{~s}, 3 \mathrm{H}), 0.09$ (s, 3H) $\delta 211.8$, $166.9,151.2,142.7,141.8,134.3,132.1,127.8,125.0,116.1,86.4,81.2,78.6,75.5,74.7$, 71.8, 55.6, 48.7, 47.4, 43.8, 34.2, 31.9, 30.2, 29.7, 29.1, 26.0, 25.8, 25.5, 22.6, 20.2, 18.3, 14.1, 12.5, 12.0, 11.0, 7.3, 5.7, -4.2, -4.3; IR (thin film, $\mathrm{CHCl}_{3}$ ) 2956, 2930, 2878, 2857, 1709, 1462, 1369, 1359, 1252, 1173, 1089, 1024, 869, 829, 774, $738 \mathrm{~cm}^{-1}$; HRMS (electrospray) for $\mathrm{C}_{45} \mathrm{H}_{80} \mathrm{O}_{8} \mathrm{Si}_{2} \mathrm{Na}[\mathrm{M}+\mathrm{Na}]^{+}$calcd. 827.5289, found 827.5301; $[\alpha]^{23}{ }_{\mathrm{D}}+13.4$ (c $\left.1.65, \mathrm{CHCl}_{3}\right)$. 


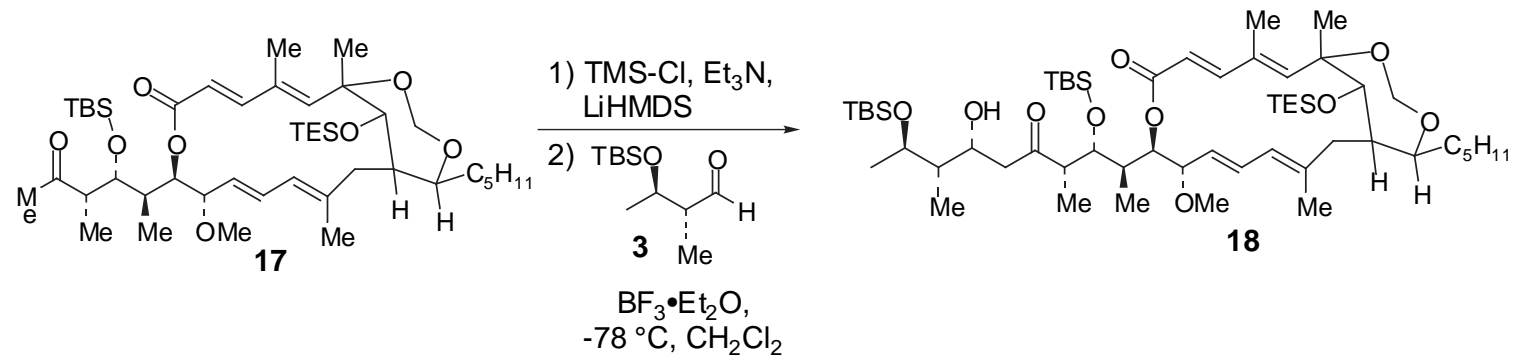

Synthesis of

$(1 S, 2 E, 4 E, 8 R, 9 S, 10 E, 12 E, 15 R, 16 S, 20 S)-8-$ $\left[\left(1^{\prime} R, 2^{\prime} R, 3^{\prime} S, 6^{\prime} R, 7^{\prime} S, 8^{\prime} R\right)\right.$-2', $7^{\prime}$-di(tert-Butyldimethylsilyl)oxy-(1',3', $7^{\prime}$-trimethyl)-non4'-one]-20-[(trimethylsilyl)oxy]-9-methoxy-1,3,13-trimethyl-16-pentyl-7,17,19trioxabicyclo[13.4.1] eicosa-2,4,10,12-tetraene-6-one (18): A solution of the methyl ketone $17(21.5 \mathrm{mg}, 0.027 \mathrm{mmol})$ in $\mathrm{THF}$ was cooled to $-78{ }^{\circ} \mathrm{C}$ and treated with a 1:1 (v:v) mixture of $\mathrm{Et}_{3} \mathrm{~N}$ and TMS-Cl $(70 \mu \mathrm{L}, 0.27 \mathrm{mmol})$, stirred for $10 \mathrm{~min}$ and then treated with LiHMDS (1.0M in THF, $70 \mu \mathrm{L}$ ). The solution was stirred for $30 \mathrm{~min}$ and then quenched by the addition of cold $\mathrm{pH} 7$ buffer then extracted with 1:1 $\mathrm{Et}_{2} \mathrm{O} /$ pentane. The organic extracts were dried over $\mathrm{Na}_{2} \mathrm{SO}_{4}$, filtered and concentrated to give $22.4 \mathrm{mg}$ of the crude enol silane, which was used in the next reaction without purification.

Freshly prepared aldehyde 3 (57 $\mathrm{mg}, 0.27 \mathrm{mmol}$ ) was azeotropically dried by concentrating with benzene (2X). A solution of the crude enol ether in benzene was then added to the aldehyde and the solution concentrated to dryness on the rotary evaporator followed by high vacuum $(3 \mathrm{~min})$. The residue was redissolved in $\mathrm{CH}_{2} \mathrm{Cl}_{2}(100 \mu \mathrm{L})$ and $4 \AA \mathrm{mol}$ sieves $(18 \mathrm{mg})$ were added. The solution stirred for $10 \mathrm{~min}$ then cooled to -78 ${ }^{\circ} \mathrm{C}$. A solution of $\mathrm{BF}_{3} \cdot \mathrm{Et}_{2} \mathrm{O}\left(80 \mu \mathrm{L}, 3.0 \mathrm{M}_{\text {in }} \mathrm{CH}_{2} \mathrm{Cl}_{2}\right)$ was added slowly down the side of the flask to allow cooling of the reagent. The reaction was stirred at $-78{ }^{\circ} \mathrm{C}$ for $40 \mathrm{~min}$ and then quenched by the addition of $\mathrm{NaHCO}_{3}$. The mixture was extracted with $\mathrm{Et}_{2} \mathrm{O}$ and the extracts were dried over $\mathrm{MgSO}_{4}$, filtered, and concentrated on the rotary evaporator 
followed by high vacuum. The residue was purified by column chromatography $(5 \mathrm{~g}$ of $\mathrm{SiO}_{2} 5 \%$ to $10 \%$ EtOAc/hexanes) to provide $23 \mathrm{mg}$ of the desired Felkin aldol that contained $10 \%$ of unreacted methyl ketone. HPLC (20 mm column, 10\% ethyl acetate/ hexanes) purification of this material then provided $19.6 \mathrm{mg}(72 \%, 80 \%$ based on recovered methyl ketone) of the desired Felkin aldol $\mathbf{1 8}$ as well as $0.4 \mathrm{mg}$ of the antiFelkin aldol diastereomer: ${ }^{1} \mathrm{H}$ NMR $\left(\mathrm{C}_{6} \mathrm{H}_{6}, 500 \mathrm{MHz}\right) \delta 7.40,(\mathrm{~d}, J=16.7 \mathrm{~Hz}, 1 \mathrm{H}), 6.64$, $(\mathrm{dd}, J=14.9,10.7 \mathrm{~Hz}, 1 \mathrm{H}), 5.92,(\mathrm{~d}, J=16.7 \mathrm{~Hz}, 1 \mathrm{H}), 5.80,(\mathrm{~d}, J=10.7 \mathrm{~Hz}, 1 \mathrm{H}), 5.77$, (s, 1H), 5.43, (dd, $J=15.1,9.3 \mathrm{~Hz}, 1 \mathrm{H}), 5.42$, (dd, $J=8.3,8.0 \mathrm{~Hz}, 1 \mathrm{H}), 4.81$, (ddd, $J=$ 9.3, 5.1, 2.4 Hz, 1H), 4.73, (d, $J=8.5 \mathrm{~Hz}, 1 \mathrm{H}), 4.68(\mathrm{dd}, J=4.6,3.2 \mathrm{~Hz}, 1 \mathrm{H}), 4.42$ (A of $\left.\mathrm{AB}, \mathrm{J}_{\mathrm{AB}}=8.6 \mathrm{~Hz}, 1 \mathrm{H}\right), 3.95,\left(\mathrm{~B}\right.$ of $\left.\mathrm{AB}, \mathrm{J}_{\mathrm{AB}}=8.6 \mathrm{~Hz}, 1 \mathrm{H}\right), 3.90-3.82,(\mathrm{M}, 2 \mathrm{H}), 3.54$, (dd, $J=9.0,6.6 \mathrm{~Hz}, 1 \mathrm{H}), 3.32,(\mathrm{~d}, J=2.2 \mathrm{~Hz}, 1 \mathrm{H}), 3.21,(\mathrm{~s}, 3 \mathrm{H}), 3.18-3.10,(\mathrm{~m}, 1 \mathrm{H}), 2.84$, (A of $\left.\mathrm{ABX}, \mathrm{J}_{\mathrm{AB}}=17.1 \mathrm{~Hz}, \mathrm{~J}_{\mathrm{AX}}=9.5 \mathrm{~Hz}, 1 \mathrm{H}\right), 2.66$, (app. q, $\left.J=7.3 \mathrm{~Hz}, 1 \mathrm{H}\right), 2.56$, (B of $\left.\mathrm{ABX}, \mathrm{J}_{\mathrm{AB}}=17.1 \mathrm{~Hz}, \mathrm{~J}_{\mathrm{BX}}=2.9 \mathrm{~Hz}, 1 \mathrm{H}\right), 2.34-2.26,(\mathrm{~m}, 1 \mathrm{H}), 1.99,(\mathrm{~s}, 3 \mathrm{H}), 1.92-1.80$, $(\mathrm{m}, 3 \mathrm{H}), 1.72,(\mathrm{~s}, 3 \mathrm{H}), 1.60-1.50,(\mathrm{~m}, 1 \mathrm{H}), 1.46(\mathrm{~s}, 3 \mathrm{H}), 1,42-1.30,(\mathrm{~m}, 7 \mathrm{H}), 1.24(\mathrm{~d}, J$ $=7.3 \mathrm{~Hz}, 3 \mathrm{H}), 1.18,(\mathrm{~d}, J=7.1 \mathrm{~Hz}, 3 \mathrm{H}), 1.16,(\mathrm{~d}, J=6.1 \mathrm{~Hz}, 3 \mathrm{H}), 1.08,(\mathrm{~s}, 9 \mathrm{H}), 0.99$, (d, $J=7.1 \mathrm{~Hz}, 3 \mathrm{H}), 0.96,(\mathrm{~s}, 9 \mathrm{H}), 0.92,(\mathrm{~s}, 9 \mathrm{H}), 0.92,(\mathrm{t}, J=8.1 \mathrm{~Hz}, 3 \mathrm{H}), 0.70-0.58,(\mathrm{~m}$, $6 \mathrm{H}), 0.34$ (s, 3H), 0.29, (s, 3H), 0.12, (s, 3H), 0.04, (s, 3H); ${ }^{13} \mathrm{C} \mathrm{NMR}\left(\mathrm{C}_{6} \mathrm{H}_{6}, 125 \mathrm{MHz}\right)$ $\delta 213.5,166.8,151.0,142.5,141.7,134.7,132.3,129.0,125.8,116.9,86,9,86.6,81.2$, $78.5,75.8,75.1,72.5,71.9,66.5,55.7,49.2,47.8,47.0,44.9,44.2,34.5,32.2,30.6,26.4$, $26.2,26.0,25.8,23.0,21.9,20.3,18.7,18.1,14.3,12.6,12.0,11.1,10.5,7.4,6.0,-3.8,-$ 4.0,-4.3, -4.9; IR (thin film, benzene) 2965, 1710, 1460, 1376, 1254, 1087, 1022, 836, 775, 737, $\mathrm{cm}^{-1}$; HRMS (electrospray) for $\mathrm{C}_{56} \mathrm{H}_{104} \mathrm{O}_{10} \mathrm{Si}_{3} \mathrm{Na}[\mathrm{M}+\mathrm{Na}]^{+}$calcd. 1043.6835, found 1043.6838; $[\alpha]_{D}^{26}+18.8(c 0.98$, benzene). 


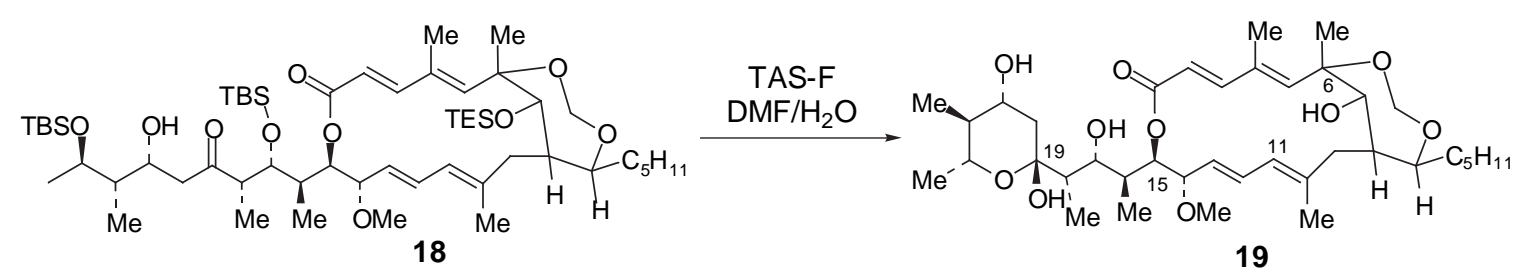

Synthes is $\quad$ of $\quad(1 S, 2 E, 4 E, 8 R, 9 S, 10 E, 12 E, 15 R, 16 S, 20 S)-8-$

$\left[\left(1^{\prime} R, 2^{\prime} R, 3^{\prime} S, 6^{\prime} R, 7^{\prime} S, 8^{\prime} R\right)-3^{\prime}-\left(2^{\prime \prime} R, 4^{\prime \prime} R, 5^{\prime \prime} R, 6^{\prime \prime} S\right)-\left(2^{\prime \prime}\right.\right.$, dihydroxy-5", $6^{\prime \prime}$-dimethyltetrahydropyran-2" -yl)-2' -hydroxy-1'-methylbutyl]-20-hydroxy-9-methoxy-1,3,13trimethyl-16-pentyl-7,17,19-trioxabicyclo[13.4.1] eicosa-2,4,10,12-tetraene-6-one

(Formamicin Aglycon) (19): A solution of TAS-F (9.7mg, $0.035 \mathrm{mmol}$,) and $\mathrm{H}_{2} \mathrm{O}(1.4$ $\mu \mathrm{L}, 0.078 \mathrm{mmol}$ ) in DMF (100 $\mu \mathrm{L})$ was added to a solution of $18(3.6 \mathrm{mg}, 0.0035 \mathrm{mmol})$ in DMF $(50 \mu \mathrm{L})$. The reaction stirred for two hours until only one lower $\mathrm{Rf}$ spot $(\mathrm{Rf}=$ 0.25) was present by TLC analysis (50\% ethyl acetate/hexanes). The reaction was concentrated to approx. $50 \mu \mathrm{L}$ and then loaded directly onto a Davisil ${ }^{\circledR}$ column (3.0 g) and eluted with a gradient of ethyl acetate/hexanes (25-50\%) to yield $2.4 \mathrm{mg}$ of a crude white solid which was further purified on HPLC (10mm column, 60\% EA/hex) to furnish $1.9 \mathrm{mg}(80 \%)$ of the formamicin aglycon 19 a white solid: ${ }^{1} \mathrm{H}$ NMR (4 : $1 \mathrm{CDCl}_{3} / \mathrm{C}_{6} \mathrm{H}_{6}$, $500 \mathrm{MHz}) \delta 7.22,(\mathrm{~d}, J=15.6 \mathrm{~Hz}, 1 \mathrm{H}), 6.48,(\mathrm{dd}, J=14.9,10.7 \mathrm{~Hz}, 1 \mathrm{H}) 5.71(\mathrm{~d}, J=$ $11.0 \mathrm{~Hz}, 1 \mathrm{H}), 5.68$ (s, 1H), 5.65, (d, $J=15.9 \mathrm{~Hz}, 1 \mathrm{H}), 5.64,(\mathrm{~d}, J=1.2 \mathrm{~Hz}, 1 \mathrm{H}), 5.15$ (dd, $J=14.9,9.5 \mathrm{~Hz}, 1 \mathrm{H}), 4.91,(\mathrm{~d}, J=9.0 \mathrm{~Hz}, 1 \mathrm{H}), 4.78,(\mathrm{~d}, J=4.1 \mathrm{~Hz}, 1 \mathrm{H}), 4.68,($ A of $\left.\mathrm{AB}, \mathrm{J}_{\mathrm{AB}}=8.7 \mathrm{~Hz}, 1 \mathrm{H}\right), 4.58,\left(\mathrm{~B}\right.$ of $\left.\mathrm{AB}, \mathrm{J}_{\mathrm{AB}}=8.7 \mathrm{~Hz}, 1 \mathrm{H}\right), 4.14,(\mathrm{~m}, J=10.7,2.7 \mathrm{~Hz}, 1 \mathrm{H})$, 3.86, ( app. t, $J=9.0 \mathrm{~Hz}, 1 \mathrm{H}), 3.70,(\mathrm{~m}, J=12.5,10.0,6.1 \mathrm{~Hz}, 1 \mathrm{H}), 3.63,(\mathrm{~m}, 1 \mathrm{H}), 3.56$, (app. t, $J=7.3 \mathrm{~Hz}, 1 \mathrm{H}), 3.51$, (dd, $J=8.3,5.6 \mathrm{~Hz}, 1 \mathrm{H}), 3.18,(\mathrm{~s}, 3 \mathrm{H}), 2.60$, (app. q, $J=$ $9.3 \mathrm{~Hz}, 1 \mathrm{H}), 2.24,(\mathrm{dd}, J=12.0,4,6 \mathrm{~Hz}, 1 \mathrm{H}), 2.14$, (ddd, $J=13.9,10.7,7.1 \mathrm{~Hz}, 1 \mathrm{H})$, 1.92, (d, $J=14.1 \mathrm{~Hz}, 1 \mathrm{H}), 1.88$, (s, 3H), $1.80-1.73$ (m, 1H), 1.77, (s, 3H), 1.69, (app. q, $J=7.1 \mathrm{~Hz}), 1.62-1.52,(\mathrm{~m}, 1 \mathrm{H}), 1.44,(\mathrm{~d}, J=5.6 \mathrm{~Hz}, 1 \mathrm{H}), 1.30-1.18(\mathrm{~m}, 8 \mathrm{H}), 1.28,(\mathrm{~s}$, $3 \mathrm{H}), 1.09,(\mathrm{~d}, J=6.4 \mathrm{~Hz}, 1 \mathrm{H}), 1.07,(\mathrm{~m}, 1 \mathrm{H}), 1.01,(\mathrm{~d}, J=7.1, \mathrm{~Hz}, 1 \mathrm{H}), 0.89,(\mathrm{~d}, J=6.6$ 
$\mathrm{Hz}, 1 \mathrm{H}), 0.86,(\mathrm{~d}, J=6.8 \mathrm{~Hz}, 1 \mathrm{H}), 0.78(\mathrm{~d}, J=6.8 \mathrm{~Hz}, 1 \mathrm{H}) ;{ }^{13} \mathrm{C} \operatorname{NMR}(4: 1$ $\left.\mathrm{CDCl}_{3} / \mathrm{C}_{6} \mathrm{H}_{6}, 125 \mathrm{MHz}\right) \delta 169.5,153.6,143.2,142.5,134.6,132.7,127.4,125.3,114.9$, $99.3,86.4,82.2,80.0,77.8,75.8,74.8,70.4,70.3,69.2,55.6,47.1,45.9,43.4,41.7,37.2$, $34.7,32.0,30.1,25.7,24.9,22.7,20.1,19.3,14.1,12.9,11.6,9.7,7.1$; IR (thin film, benzene) $3419,2929,1682,1455,1260,1150,1087,1037,968,899,847,808,678 \mathrm{~cm}^{-1}$; HRMS (FAB) for $\mathrm{C}_{38} \mathrm{H}_{62} \mathrm{O}_{10} \mathrm{Na}[\mathrm{M}+\mathrm{Na}]^{+}$calcd. 701.4241, found 701.4244; $[\alpha]^{26}{ }_{\mathrm{D}}+12.1$ (c 0.19 , benzene). 


\section{STEREOCHEMICAL ASSIGNMENTS FOR COMPOUNDS 9 AND 22}

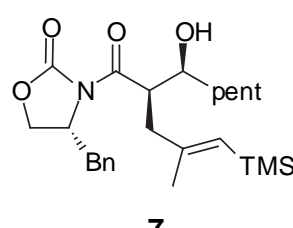

7

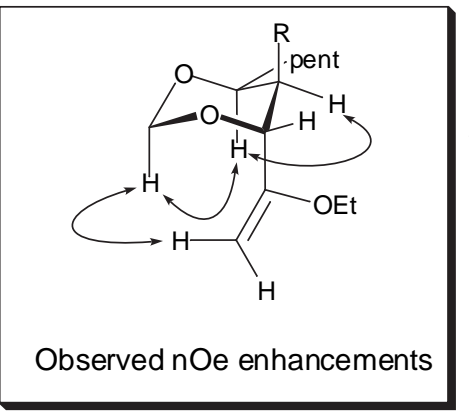

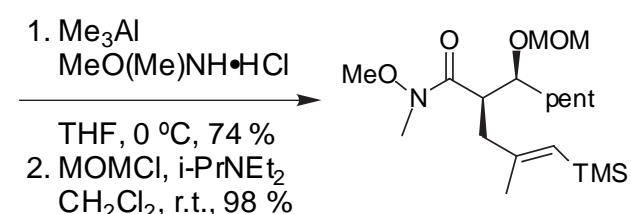

24

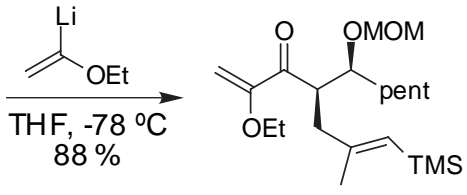

25

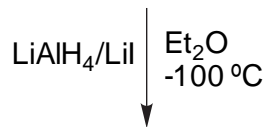

1. Separation

$\frac{\text { 2. } \mathrm{Me}_{2} \mathrm{BBr}, 2,6-\mathrm{DTMP}}{\mathrm{CH}_{2} \mathrm{Cl}_{2},-78{ }^{\circ} \mathrm{C}}$

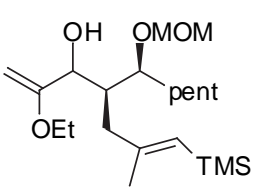

9-syn $/ 9-a n t i=1 / 1$

Aldol 7 was converted to the $\beta$-methoxymethoxy Weinreb amide 24 in 2 steps (73\%). Displacement of the amide moiety by $\alpha$-ethoxyvinyllithium in THF, followed by nonselective reduction of the ketone function afforded a 1/1 mixture of 9-syn and 9-anti. Formation of the cyclic acetal 26 was effected by treatment with $\mathrm{Me}_{2} \mathrm{BBr}$ and 2,6-DTMB. NOe experiments on the acetal derived from 9-syn were not conclusive. However, nOe enhancements were observed for $\mathbf{2 6}$ (derived from 9-anti) between the $\mathrm{C}(1)$ and acetal proton, $\mathrm{C}(4)$ and $\mathrm{C}(5)$ proton as well as $\mathrm{C}(5)$ and acetal proton, indicating an anti relationship between $\mathrm{C}(3)$ and $(\mathrm{C} 4)$ proton and a syn relationship between $\mathrm{C}(4)$ and $\mathrm{C}(5)$ proton. By deduction, compound 9-syn possess a syn,syn relationship between the $\mathrm{C}(3)$, $\mathrm{C}(4)$ and $\mathrm{C}(5)$ stereocenters. 


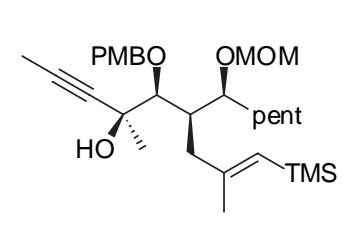

22

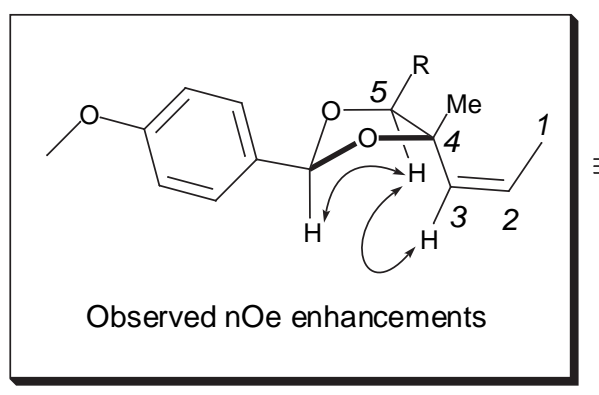

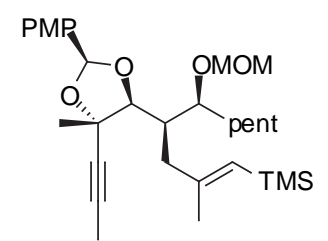

27

$\mathrm{H} 2$

Lindlar's catalyst

Quinoline, EtOAc

r.t., $78 \%$

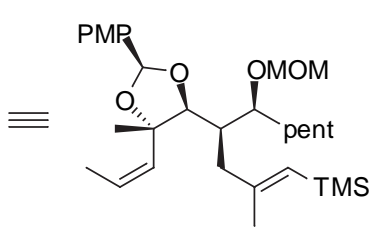

28

DDQ oxidation of the PMB ether 22 gave the p-methoxylidene (PMP) acetal 28.

Semi-hydrogenation of the triple bond with $\mathrm{H}_{2}$ and Lindlar's catalyst afforded 29. Key ${ }^{1} \mathrm{H}$ nOe enhancements were observed between the $\mathrm{C}(3), \mathrm{C}(5)$ and acetal methine proton, indicating a syn relationship for the $\mathrm{C}(4)-\mathrm{C}(5)$ hydroxyl functions in $\mathbf{2 2}$. 
Table 1: ${ }^{1} \mathrm{H}-\mathrm{NMR}$ and ${ }^{13} \mathrm{C}-\mathrm{NMR}$ Data of formamicin and formamicin aglycon.

\begin{tabular}{|c|c|c|c|c|c|c|}
\hline Position & Formamicin & $\begin{array}{l}{ }^{13} \mathrm{C}-\mathrm{NMR} \\
\text { Formamicin } \\
\text { aglycon }\end{array}$ & Difference & Formamicin & $\begin{array}{l}{ }^{1} \mathrm{H}-\mathrm{NMR} \\
\text { Formamicin } \\
\text { Aglycon }\end{array}$ & Difference \\
\hline 1 & 169.6 & 169.5 & 0.1 & & & 0.00 \\
\hline 2 & 115.0 & 114.9 & 0.1 & 5.69 & 5.65 & 0.04 \\
\hline 3 & 153.8 & 153.6 & 0.2 & 7.27 & 7.22 & 0.05 \\
\hline 4 & 134.6 & 134.6 & 0.0 & & & 0.00 \\
\hline 5 & 142.6 & 142.5 & 0.1 & 5.71 & 5.68 & 0.03 \\
\hline 6 & 80.0 & 80.0 & 0.0 & & & 0.00 \\
\hline \multirow[t]{2}{*}{7} & 74.8 & 74.8 & 0.0 & 3.56 & 3.51 & 0.05 \\
\hline & & & & 1.66 & 1.58 & 0.08 \\
\hline 8 & 47.2 & 47.1 & 0.1 & 2.65 & 2.60 & 0.05 \\
\hline \multirow[t]{2}{*}{9} & 34.8 & 34.7 & 0.1 & 1.83 & 1.78 & 0.05 \\
\hline & & & & 1.97 & 1.92 & 0.05 \\
\hline 10 & 143.4 & 143.2 & 0.2 & & & 0.00 \\
\hline 11 & 125.3 & 125.3 & 0.0 & 5.76 & 5.71 & 0.05 \\
\hline 12 & 132.9 & 132.7 & 0.2 & 6.53 & 6.48 & 0.05 \\
\hline 13 & 127.8 & 127.4 & 0.4 & 5.20 & 5.15 & 0.05 \\
\hline 14 & 82.3 & 82.2 & 0.1 & 3.91 & 3.86 & 0.05 \\
\hline 15 & 75.8 & 75.8 & 0.0 & 4.96 & 4.91 & 0.05 \\
\hline 16 & 37.3 & 37.2 & 0.1 & 2.19 & 2.14 & 0.05 \\
\hline \multirow[t]{2}{*}{17} & 70.5 & 70.4 & 0.1 & 4.20 & 4.14 & 0.06 \\
\hline & & & 0.0 & 4.87 & 4.78 & 0.09 \\
\hline 18 & 41.9 & 41.7 & 0.2 & 1.76 & 1.69 & 0.07 \\
\hline 19 & 99.4 & 99.3 & 0.1 & 5.71 & 5.64 & 0.07 \\
\hline \multirow[t]{2}{*}{20} & 40.2 & 45.9 & -5.7 & 1.17 & 1.07 & 0.10 \\
\hline & & & & 2.34 & 2.24 & 0.10 \\
\hline \multirow[t]{2}{*}{21} & 76.2 & 69.2 & 7.0 & 3.81 & 3.63 & 0.18 \\
\hline & & & 0.0 & & 1.44 & $21(\mathrm{OH})$ \\
\hline $22^{\mathrm{a}}$ & 43.7 & 43.4 & 0.3 & $1.20-1.35$ & $1.18-1.30$ & 0.04 \\
\hline 23 & 69.8 & 70.3 & -0.5 & 3.78 & 3.70 & 0.08 \\
\hline 24 & 19.5 & 19.3 & 0.2 & 1.15 & 1.09 & 0.06 \\
\hline 25 & 78.0 & 77.8 & 0.2 & 3.61 & 3.56 & 0.05 \\
\hline $26^{\mathrm{a}}$ & 30.2 & 30.1 & 0.1 & $1.20-1.35$ & $1.18-1.30$ & 0.04 \\
\hline $27^{\mathrm{a}}$ & 25.8 & 25.7 & 0.1 & $1.20-1.35$ & $1.18-1.30$ & 0.04 \\
\hline $28^{\mathrm{a}}$ & 32.0 & 32.0 & 0.0 & $1.20-1.35$ & $1.18-1.30$ & 0.04 \\
\hline $29^{a}$ & 22.8 & 22.7 & 0.1 & $1.20-1.35$ & $1.18-1.30$ & 0.04 \\
\hline 30 & 14.2 & 14.1 & 0.1 & 0.91 & 0.86 & 0.05 \\
\hline 31 & 11.7 & 11.6 & 0.1 & 1.83 & 1.77 & 0.06 \\
\hline 32 & 24.9 & 24.9 & 0.0 & 1.33 & 1.28 & 0.05 \\
\hline 33 & 86.5 & 86.4 & 0.1 & 4.63 & 4.58 & 0.05 \\
\hline 34 & 20.2 & 20.1 & 0.1 & 1.93 & 1.88 & 0.05 \\
\hline 35 & 55.7 & 55.6 & 0.1 & 3.23 & 3.18 & 0.05 \\
\hline 36 & 9.7 & 9.7 & 0.0 & 0.83 & 0.78 & 0.05 \\
\hline 37 & 7.3 & 7.1 & 0.2 & 1.08 & 1.01 & 0.07 \\
\hline 38 & 13.3 & 12.9 & 0.4 & 0.99 & 0.89 & 0.10 \\
\hline
\end{tabular}

${ }^{a}$ The proton resonances overlapped and could not be resolved. 